\title{
Die Psalmen in gotischer Sprache
}

\author{
Arend Quak \\ Instituut voor Oudgermanistiek/Scandinavisch Seminarium, Universiteit \\ van Amsterdam, Amsterdam, Niederlande \\ a.quak@uva.nl
}

\begin{abstract}
Although the text of the psalms did not survive in Wulfila's Gothic bible translation, some verses are cited in the bible fragments and in other Gothic texts. Here these quotes are compared with surviving West-Germanic translations of the same passages to view the differences and the similarities between them.
\end{abstract}

\section{Keywords}

Gothic psalms - Old English psalters - Notker Teutonicus - Old Dutch psalter

\section{$1 \quad$ Einleitung}

Die Psalmen gehören zu den wichtigen religiösen Texten im Mittelalter. Vor allem im späten Mittelalter wurden sie wichtig in der Schule, vgl. Blom 2017:3738. Aber auch früher schon hatten sie große Bedeutung. Nach der 'Admonitio generalis' Karls des Großen von 789 sollte man in der Schule religiöse Musik, Singen und die Psalmen unterrichten, damit man den Glauben verbreiten könne. Es ist daher kein Zufall, dass die Psalmen zu den ersten Texten gehörten, die man in Nordwesteuropa im 8. und 9. Jahrhundert in die Volkssprachen übertrug. Solche Übersetzungen finden sich im Altenglischen, Althochdeutschen, Altsächsischen, Altfriesischen und Altniederländischen. Auch die Goten dürften eine Psalmenübersetzung gehabt haben, denn es wird gesagt, dass Wulfila auch das Alte Testament wenigstens teilweise übersetzt habe. Die gotische Bibel ist zwar mehrere Jahrhunderte älter als die westgermanischen Texte, aber es dürfte interessant sein zu sehen, welche Terminologie Wulfila in der Übersetzung der Psalmen gebrauchte und sie mit der Terminologie in den 
ältesten Übertragungen in Nordwesteuropa zu vergleichen. Allerdings haben wir Wulfilas Text nicht in direkter Überlieferung. Nach Auxentius hat er zwar die ganze Bibel übersetzt - mit Ausnahme der Bücher der Könige, weil die vielen Kämpfe darin ein schlechtes Beispiel für seine gotischen Schäflein seien -, aber vom Alten Testament blieb nur ein Fragment aus 'Nehemia' erhalten. Obwohl auch das Neue Testament nicht vollständig erhalten ist, finden sich dort Zitate aus den Psalmen und außerdem sind Psalmzitate auch in anderen Texten überliefert. Teile der gotischen Übersetzung der Psalmen lassen sich in folgenden Quellen finden:

1. Das Fragment von Bologna ist ein gotischer Text, der 2010 als scriptio inferior unter einem Fragment von Augustins 'De civitate Dei' entdeckt wurde. Der Text wurde zuerst 2013 von Finazzi \& Tornaghi mit italienischem Kommentar herausgegeben und erschien dann 2014 auf Deutsch mit Ergänzungen und Berichtigungen von Carla Falluomini, die dann 2017 noch einen Nachtrag veröffentlichte. Es handelt sich bei diesem Fragment um den Rest eines Doppelblattes, das vermutlich aus einer Predigt stammt, die sehr viele Zitate aus dem Alten und Neuen Testament enthält. Zu diesen Zitaten gehören auch elf Zitate aus den Psalmen, von denen Falluomini (2014: 294) wohl mit Recht annimmt, dass sie auf die Übersetzung von Wulfila zurückgehen. Sie gibt in ihrem Beitrag von 2017 den gotischen Text mit den vermutlichen griechischen Entsprechungen, auf den hier verwiesen und nach dem hier zitiert wird.

2. Im Jahre 2015 entdeckten Andrej Vinogradov und Maksim Korobov, dass einige bis dahin unidentifizierte Inschriften aus der Basilika von Mangup auf der Krim in gotischer Sprache abgefasst waren. Eine dieser Inschriften ist ein Zitat aus den Psalmen. Sie wird hier zitiert nach der Ausgabe der beiden russischen Forscher (2016).

3. Die dritte Quelle bilden Zitate der Psalmen im Neuen Testament. Für die Juden im ersten Jahrhundert war das Alte Testament die Heilige Schrift und diese war wichtig im täglichen Leben: "Among the various texts from the Old Testament the Book of Psalms, ..., enjoyed a special position. As a collection of prayers, hymns, laments and similar poetic genres, it was capable of giving expression to various religious experiences" (Moyise \& Menken 2004: 1). Im Neuen Testament wird insgesamt 116 Male an die Psalmen referiert. Auch in Wulfilas Übersetzung der Bibel finden sich selbstverständlich Zitate aus den Psalmen. An einer Stelle wird das sogar ausdrücklich gesagt. In Eph. 4,8 (Handschrift A) findet sich die Randglosse psalmo, die darauf hinweist, dass der darauf folgende Text ussteigands in hauhipa ushanp hunp jah atuhgaf gibos mannam auf Ps. 67,18 ASCENDISTI IN ALTUM CEPISTI CAPTIVITATEM ACCEPISTI DONA 
IN HOMINibUs zurückgeht. Zitate aus den Psalmen werden manchmal ausdrücklich eingeleitet: Im Fragment von Bologna wird etwa gesagt, dass "der Prophet", i.c. David, zitiert wird: bi hvana qap praufetus 'über wen der Prophet sagte'. Im neuen Testament wird meistens nur gesagt, dass es geschrieben ist: swaswe ist gamelip (s. unten Nr. 21, 23 und 27). Aber man findet auch mehr spezifische Hinweise auf das Gesetz: ei usfullnodedi waurd pata gamelido in witoda ize 'damit das Wort erfüllt werden möge, das in ihrem Gesetz geschrieben wurde' (Nr. 18), niu ist gamelip in witoda izwaramma 'ist nicht geschrieben in eurem Gesetz?' (nr. 22) und nih pata gamelido ussuggwup habt ihr nicht in der Schrift gelesen' (Nr. 28). Sogar der Prophet David wird hier einmal namentlich genannt: jah silba Daweid qipip in bokom psalmo〈no 'und David selbst sagt im Buch der Psalmen' (Nr. 26). Allerdings ist Wulfilas Text nicht vollständig überliefert. Insgesamt finden sich 34 Zitate bzw. Anspielungen in den Handschriften, die sich bis heute erhalten haben, ${ }^{1}$ cf. Moyise \& Menken 2004: 247-48. Manche dieser Zitate erscheinen mehr als einmal im Material, vgl. etwa pu is sunus meins sa liuba aus Ps. 2,7: Dominus DIXIT AD ME: FILIUS MEUS ES TU, erscheint in Mk. 1,11, Mk. 9,7 und Luk. 3,22 in ähnlicher Form. In anderen Fällen wird der Text der Psalmen nicht buchstäblich zitiert, sondern es wird darauf angespielt, z.B. in Luk. 4,6-8, findet man Reminiszenzen von Ps. 2,8. Solche Stellen wurden hier vorläufig außer Betracht gelassen.

Obwohl die Psalmen somit nur spärlich im Gotischen überliefert sind, kann es doch interessant sein zu sehen, welche Übersetzungen für bestimmte Termini Wulfila gewählt hat und welche die späteren Übersetzer in Nordwesteuropa bevorzugten. Man soll dabei allerdings berücksichtigen, dass der gotische Bischof aus dem Griechischen übertrug und seine Kollegen in Nordwesteuropa im 8. und 9. Jahrhundert von der lateinischen Version der Bibel ausgingen, ob das nun das 'Psalterium Romanum' ist wie in den frühen altenglischen Psaltern, oder das 'Psalterium Gallicanum' wie in den kontinentalen und in den jüngeren altenglischen Übertragungen. Es ist unsicher und sehr umstritten, ob Wulfila auch die lateinische Übertragung, die Vetus Latina, benutzt hat, obwohl er nach Auxentius sowohl im Griechischen wie im Lateinischen bewandert war. Wohl ist es wahrscheinlich, dass der lateinische Text den gotischen Kopisten der Handschriften im Italien des 5. und 6. Jahrhunderts zugänglich war, da sie in einer lateinischen katholischen Umgebung arbeiteten. Die Basis

1 z. B. in der Apostelgeschichte finden sich 15 Zitate aus dem Buch der Psalmen, aber dieser Teil des Neuen Testaments ist im Gotischen nicht erhalten, obwohl er vermutlich wohl existierte, da das Fragment von Bologna verweist auf in tojam apaustaule 'in den Taten der Apostel'. 
für die volkssprachige Übersetzung war jedoch in allen Fällen eine germanische Sprache und es dürfte interessant sein zu sehen, welche Termini Wulfila wählte und welche seine späteren Kollegen. Das Gotische war ja seit dem 1. Jahrhundert seinen eigenen Weg gegangen und dürfte außerdem auf dem Weg durch Osteuropa Einflüsse aus anderen Sprachen erfahren haben. Es hatte sich im 4. Jahrhundert erheblich von den anderen germanischen Sprachen hinweg entwickelt, wie man erkennen kann, wenn man die nordwestgermanische Runeninschrift von Gallehus (ca. 40o) mit dem Text ek Hlewagastiz holtijaz horna tawido 'ich Hlewagast, der Sohn des Holte (?), machte das Horn' mit einer Rekonstruktion desselben Textes in der Sprache Wulfilas vergleicht: * $i k$ Hliugasts hulteis haurn tawida.

Das Altenglische hat von allen frühgermanischen Sprachen die reichste Überlieferung der Psalmen in der Volkssprache. Nicht weniger als 13 mehr oder weniger vollständige Übersetzungen aus dem 8. bis 12. Jahrhundert sind überliefert. Die Prosapsalmen (Ps. 1-50) in einer Pariser Handschrift dürften dabei die Kopie eines Textes aus dem späten 9. Jahrhundert aus Wessex sein, der möglicherweise von König Alfred übersetzt wurde (O'Neill 2016: ix). In diesem Beitrag werden folgende Psalter berücksichtigt: Vespasian [ca. 875-900] ${ }^{2}$ in der Ausgabe von Kuhn; Junius [ca. 925] in der Ausgabe von Brenner; Regius [um 950] in der Ausgabe von Roeder; Bosworth [ ${ }^{\left.2975^{-1000}\right]}$ in der Ausgabe von Lindelöf; Cambridge [um 1025] in der Ausgabe von Wildhagen; Lambeth [ca. 1025] in der Ausgabe von Lindelöf; Vitellius [ca. 1050] in der Ausgabe von Rosier; ${ }^{4}$ Arundel [ca. 1050-75] in der Ausgabe von Oess; Stowe [1050-75] in der Augabe von Kimmens, Tiberius [1050-1075] in der Ausgabe von Campbell, Salisbury [1100] in der Ausgabe von Sisam \& Sisam und Canterbury [1150] in der Ausgabe von Harsley. Auch wurde der Text in der Pariser Handschrift BN $8824[ \pm 1050]$ in der Ausgabe von O'Neill herangezogen, obwohl er deutlich freier ist als die anderen Übertragungen. Für die ersten 5 o Psalmen wurde auch die Ausgabe von Pulsiano (2001) herangezogen.

Auf dem Kontinent ist die Zahl der erhaltenen Psalmübertragungen weit geringer. Für das Althochdeutsche hat man im Grunde nur die Übersetzung von Notker Teutonicus (Anfang des 11. Jhs.), die hier in der Ausgabe von Petrus Tax herangezogen wurde. Sonst finden sich nur Fragmente wie die alemannischen

2 Diese und die anderen Datierungen wurden aus Sisam \& Sisam 1959 übernommen.

3 Durch den fragmentarischen Charakter erscheint diese Handschrift nur einmal in den unten zitierten Texten.

4 Die Ausgabe wird von Berghaus (1979: 15) als "fehlerhaft" eingestuft. 
Psalmen und der bairische Psalm $138 .{ }^{5}$ Sie haben jedoch keine Entsprechungen im gotischen Text. Daher werden hier auch zwei frühmittelhochdeutsche Psalmenübersetzungen herangezogen: Vindobonensis (Kirchert 1979) und Windberg (Törnqvist 1934). Die 'Wachtendonckschen Psalmen' in der Ausgabe von de Grauwe (1979-82) unter Heranziehung von Quak (1981) wurden für das Altniederländische berücksichtigt. Als Vergleich wird ab und zu auch der älteste mitelniederländische Text herangezogen: Leningrad (Heymans 1973). Fürs Altsächsische stehen die 'Lubliner Psalmen' in der Ausgabe von Tiefenbach (2003) Modell. ${ }^{6}$

Im Folgenden werden die einzelnen in gotischer Sprache überlieferten Psalmenstellen behandelt und nach Wortschatz und Parallelen in anderen germanischen Sprachen kommentiert. Ausgangspunkt bildet der lateinische Text der Psalmen, da er den westgermanischen Übertragungen zurundeliegt.

1. Ps. 2,7: Filius meus es tU, Ego hodie genui te. 'Du bist mein Sohn, heute habe ich dich geboren'. Dieser Psalmvers wird zitiert in Mk. 1,11: pu is sunus meins sa liuba, in puzei waila galeikaida. ( $\sigma \dot{v}$ घi $\delta$ viós $\mu \circ v$

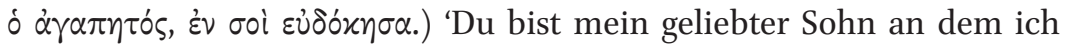
mich erfreue'. Genau derselbe Text wird zitiert in Luk. 3,22. In Mk. 9,7 erscheint der Text leicht modifiziert: sa ist sunus meins sa liuba. Der gotische Text unterscheidet sich kaum von den jüngeren Übertragungen, nur dass sa liuba im Text steht, was sich aber aus dem griechischen Text erklären lässt. Die weitgehende Übereinstimmung in der Wortwahl zwischen den germanischen Texten liegt nahe, da es sich um allgemeine Bezeichnungen handelt. Der altniederländische Text (10. Jh.) etwa hat fast genau denselben Text: sun min bis thu, ik hiudo gebar thik. Notker hat praktisch denselben Text: min sun bist tu. híuto gebár ih tih; das ist auch der Fall in Windberg und im Vind. Auch im Altenglischen werden fast dieselben Wörter benutzt:

5 Abgedruckt in Steinmeyers 'Kleineren althochdeutschen Sprachdenkmälern' (1916).

6 Es gibt noch ein Fragment einer altsächsischen Psalmenübersetzung aus Paderborn (Quak 1988) und den Psalmenkommentar von Gernrode (in: Wadstein 1899), aber die dort überlieferten Verse decken sich nicht mit den im Gotischen überlieferten. Dasselbe gilt für die altfriesischen Fragmente (vgl. Langbroek 1990). 
sunu min ðu earð ic to dege ic cende ðec (Vesp.);

sunu min pu eart ic to drege ic cende đe (Junius); sunu eart ðu ic to daeg cende [ ] (Regius); ${ }^{7}$

sunu min eart pu ic to doege cende pe (Cambridge);

sunu min pu eart ic to dceg gestrynde pe (Lambeth);

cild min pu eart ic [ ] doege acende pe (Vitellius);

bearn min eart pu ic todcege accende pe (Arundel);

sunu min eart ðu ic todoeg cende pe (Stowe);

sunu min eart pu ic todceg caende pe (Tiberius);

sunu min eart pu ic todceg cende pe (Salisbury);

min sunu eart ðu ic todceg pe acende (Canterbury);

Pu eart min sunu; nu todaeg ic ðe acende (Paris).

Im Grunde haben alle Übersetzungen denselben Text mit nur wenigen Ausnahmen. In Vitellius findet sich cild 'Kind' und in Arundel findet sich bearn 'Kind' statt sunu 'Sohn'. Für das Verb im zweiten Teil benutzt nur Lambeth gestrynde zum Verb gestreónan 'gewinnen' (вт 928) statt wie alle anderen (a)cennen 'zeugen' (вт 150) für genui. ${ }^{8}$

2. Ps. 3,9: DOMINI EST SALUS ET SUPER POPULUM TUUM BENEDICTIO TUA. Dieser Vers erscheint als fraujins ist naseins jah ana managein peinai piupeins peina. 'des Herrn ist die Rettung und über dein Volk dein Segen' im Fragment Bologna Bl. Iv, Z. 19f. Für 'Rettung' benutzt das Gotische hier das Substantiv naseins. Ableitungen von der Wurzel pgm. "naz- 'retten' erscheinen regelmäßig für lat. salus 'Rettung' und damit verwandte Wörter, vgl. etwa anl. neriando 'Retter, Heiland' und nerien 'retten'. Bei Notker findet man die Wurzel pgm. *hail-: heili 'Rettung': Târ schînet daz cótes diû hêili ist. [...], obwohl nerien 'retten' auch im Althochdeutschen vorkommt. Auch im Altenglischen erscheint dieselbe Wurzel: hcel 'Rettung' (вт 499-500) bzw. hcelu 'id' (вт 500). Im zweiten Teil des Verses ist im Gotischen das Substantiv piupeins eine der möglichen Übertragungen von gr. eulogía, vgl. auch II Cor. 9,6.6 und Eph. 1,3. Es gehört zusammen mit dem Verb piupjan 'segnen', dem Substantiv piupida 'der Gesegnete' und dem Adjektiv piupeigs 'gesegnet', die zu einer Wurzel mit der Bedeutung 'gut' gehören, vgl. Lehmann 1986: 362-63. Das Gotische scheint hier eine eigene Terminologie entwickelt zu haben, denn in den anderen germanischen Sprachen erscheint die

7 Im Folgenden wird mit [ ] angegeben, dass die Übersetzung von Teilen des lateinischen Textes fehlt.

8 Auch der mittelniederländische Psalter von Leningrad hat hier gewonnen. 
Wurzel selten. Im Altnordischen findet sich pýðr 'freundlich' und pýða 'freundlich stimmen, zum Freunde machen', weiter findet sich noch ae. gepiede 'gut, tugendhaft' (вт 485) mit dem Verb gepîedan 'anschließen, verbinden, vereinen' und es ist möglich, dass as. githiudo 'auf gebührende Weise' auch zu dieser Gruppe gehört, vgl. Kroonen 2013: 539. Die Wörter ahd. gidiuti (Adj.) 'volkssprachlich' und gidiuto (Adv.) 'öffentlich', die oft in diesem Zusammenhang zitiert werden - z.B. in Lehmann 1986 -, werden in EWA (IV,236-37 und 237) wahrscheinlich mit Recht wegen ihrer Bedeutung von dieser Wurzel getrennt: Sie gehören zur Wurzel *thiod'Volk', vgl. Orel 2003: 423. Es sieht also danach aus, dass das Gotische hier eine alte Wurzel bevorzugte, die sporadisch auch in den anderen germanischen Sprachen erscheint. Es ist wohl kein Zufall, dass dieselbe Wurzel auch in Ps. 117,26 erscheint: piupida sa qimanda in namin fraujins! 'gesegnet ist derjenige, der im Namen des Herrn kommt' (s. unten Nr. 29). Die WPs. benutzen in Ps. 3,9 für lat. benedictio das Substantiv geuuîunga, ${ }^{9}$ und in Notkers Übersetzung findet man segen 'Segen': Vnde dîn ségen is uber dînen liût. Die beiden frühmittelhochdeutschen Texte haben fast denselben Text wie Notker: Gotes ist daz heil un [de] ûber daz livte din der segen din (Vind.) und Des herren ist daz heil und uber liut dinen wihe oder segen din (Windb.). Im Altenglischen erscheinen:

dryhtnes is heelu 7 ofer folc ðin bledsung Jin. (Vesp.); dryhtnes is hcelu 7 ofer folc pin bledsung din (Junius); dryhtnes is hcelo 7 ofer folc [ ] bledsung pin (Regius); drihtyn is hcelu 7 ofyr folc pin bletsung pin (Cambridge); drihtnes is heel 7 ofor folc pin pin bletsung (Lambeth); drihtnes is heelo 7 ofer folc pin bletsung pin (Vitellius); drihtnes is heelo 7 ofer folc pin bletsung pin (Arundel); drihtne is hcel 7 ofer folce ðine bletsung Jin (Stowe); drihten is heelo 7 ofer folc pin bletsuncg pin (Tiberius); drihten is hoel 7 ofer fol pin bletsung pin (Salisbury); Drihtnes is hęlo 7 ofer pin folc pin bletsung (Canterbury); for Jam on ðe ys eall ure hoel and ure tohopa and ofer pin folc sy pyn bletsuncg (Paris). ${ }^{10}$

Nach de Grauwe (1979: § 8) war das Verb (gi)wîhan auf dem Kontinent wohl die älteste Wiedergabe von lat. benedicere und wurde es dort später durch das Lehnwort seganon ersetzt. Das Englische hat von Anfang an bletsian.

10 Hier und auch später wird deutlich, dass sich der Text der Prosapsalmen (Ps. 1-50) den anderen Psaltern zwar folgt, aber viel freier übersetzt. In den metrischen Psalmen ist er bekanntlich noch viel freier. 
Hier wird also in allen altenglischen Psalmübertragungen die spezifisch altenglische Bezeichnung bletsung 'Segen' (вт 109) für benedictio benutzt. Auch sonst sind die Übertragungen mit Ausnahme des Pariser Psalters fast identisch.

3. Ps. 4,5: IRASCIMini, ET NOlite PECCARE 'werdet nicht böse und sündigt nicht'. Dieser Psalmvers wird zitiert als pwairhaippan sijaip jah ni frawaurkjaip 'ihr sollt nicht böse sein und ihr sollt nicht sündigen' in Eph. 4,36, sowohl in Handschrift A als in B. Das Wort, das hier im gotischen Text für 'böse' verwendet wird, ist pwairhs. Dieselbe Bedeutung erscheint auch in ae. pweorh 'cross, angry, bitter' (BT 1083), aber normalerweise bedeutet dieses Adjektiv 'quer' (Kroonen 2013: 556), wie etwa im Althochdeutschen, Altsächsischen und Altnordischen, cf. Orel 2003: 432. Die Bedeutung 'böse, erzürnt' dürfte eine jüngere Entwicklung sein. Im Gotischen erscheint auch das Substantiv pwairhei 'Bosheit, Wut' regelmäßig. Notker hat in dieser Stelle: Pélgent iûuih mit dem Verb belgan 'zürnig sein, werden', das das normale Wort für lat. irasci im Westgermanischen zu sein scheint, s. de Grauwe 1979-81: §115. Das Verb findet sich nicht im Gotischen. ${ }^{11}$ Eine Ableitung derselben Wurzel findet sich allerdings in got. balgs 'Schlauch'. Wenn man annimmt, dass die ursprüngliche Bedeutung von pie. ${ }^{*} b^{h} e l \hat{g}^{h_{-}}$'(an)schwellen' war (s. Kroonen 2013: 49, 58 ), hat sich diese erst später und nur im WGerm. zu 'zürnen' entwickelt. Die Situation ist somit etwa umgekehrt zu dem oben beschriebenen Bedeutungswandel in got. pwairhs 'zornig' < 'quer' (im WGerm. erhaltengeblieben). Andere kontinentale Texte bevorzugen das Verb zürnen: Zurnet un[de] nieht ensuntet (Vind.) und Erzurnet unde newellent sunten (Windb.). ${ }^{12}$ Die altenglischen Texte benutzen an dieser Stelle das Verb irsian böse werden, zürnen' (вт 6оо) und sind sich dabei sehr einig:

eorsiað 7 nyllað syngian (Vesp.);

eorsiad 7 nyllad syngian (Junius);

eorsiað [ ] nellen ge syngian (Regius);

yrsiað 7 nellað syngian (Cambridge);

yrsiað 7 nelle ge syngian (Lambeth);

iersiað ge 7 nellan ge syngian (Vitellius);

irsiap 7 nellan syngian (Arundel);

eorsiað 7 nylle ge singian (Stowe);

fehlt (Tiberius);

11 Nach EWA I,528-29 erscheint die Wurzel in allen germanischen Sprachen mit Ausnahme des Gotischen, aber zeigt eine Tendenz in diesen Sprachen zu verschwinden.

Vgl. der mittelniederländische Leningrader Psalter: Tornet ende ne zondicht niet. 
yrsiað 7 nelle ge na synnian (Salisbury);

yrsyað 7 nellen ge sengien (Canterbury)

Peah hit gebyrige pret ge on woh yrsien, ne scule ge hit no py hrapor purhteon, pe laes ge syngien (Paris).

Alle altenglischen Texte übersetzen also lat. peccare mit ae. syngian 'sündigen' (вт 965). Für diesen Begriff verwendet das Gotische frawaurkjan, ein Verb, das u.a. im Althochdeutschen mit ähnlicher Bedeutung erscheint: farwirken 'verschulden, verwirken' (Schützeichel 2006: 421). Im Althochdeutschen und Altsächsischen ist die übliche Übertragung für lat. peccare das mit dem altenglischen Wort verwandte Verb (gi)sunton 'sündigen' (vgl. Köbler 1975) oder eine Ableitung davon. Hier gehen also die westgermanischen Sprachen zusammen, denn auch das Altniederländische (sunda 'peccatum', sundige 'peccatores') und das Altfriesische (sendigia 'sündigen' - Hofmann-Popkema 2008: 421) schließen sich an.

4. Ps. 6,9: Discedite a me omNes QUi operamini iniquitatem 'weichet von mir alle, die ihr Frevel tut'. In Matt. 7,23 findet man dafür: afleipip fairra mis, jus waurkjandans unsibjona 'geht von mir weg, ihr die ihr Gottlosigkeit pflegt'. In unsibjona erscheint der Akk. Pl. ntr. des Adjektivs unsibjis 'rechtlos, gottlos', cf. Ps. 11,9 unten (Nr. 7). Für 'weggehen' benutzt das Gotische hier afleipan 'weggehen, sich entfernen' zum starken Verb pgm. *lîpan 'gehen' in Kombination mit dem Adverb fairra 'fern'. Dieses Verb erscheint auch regelmäßig in den anderen germanischen Sprachen mit derselben Bedeutung, aber es wird dort nicht an dieser Stelle verwendet. Im Altenglischen findet man hier:

gewitað from me alle ða wircað unrehtwisnisse (Vesp.);

gewitad from me ealle pe wyrcead unryhtwisnesse (Junius);

gewitað fram me ealle pe wyrcað unryhtwisnesse (Regius);

gewitað from me ealle pe wyrceað unrihtwisnysse (Cambridge);

gewitap fram me ealle ge pe wyrcað unrihtwisnesse (Lambeth); onweg gewitað from me ealle pa pe wyrceað unrihtwisnesse (Vitellius); onweg gewitap fram me ealle pa pe wircap unrihtwisnesse (Arundel); gewitað fram me ealle pa pe wyrcap unrihtwisnysse (Stowe); gewitað fram me ealle pe wyrcap unrihtwiswessa (Tiberius); gewitap fram me ealle pa wircap unrihtwisnesse (Salisbury); gewitce from me ealle pce pe unriht wirchað (Canterbury); Gewitað fram me ealle pa pe unriht wyrcað (Paris).

Alle altenglischen Psalter benutzen somit das Verb gewittan 'gehen, weggehen' (вт 469), das in dieser Bedeutung wohl spezifisch altenglisch ist, vgl. 
Kroonen 2013:589. Notker hat hier: Skêident iûuih fóne mir alle úbel-tâtige. Die beiden frühmittelhochdeutschen Texte benutzen wichen (Vind.) bzw, entwichen (Windb.). Das Verb pgm. *wurkjan 'tun, verrichten' erscheint in mehreren germanischen Sprachen in derselben Bedeutung und sogar

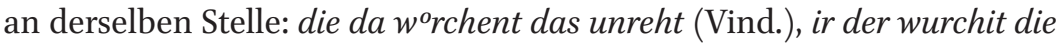
unrechticheit (Windb.). Es ist die nullstufige Präsensform, die zur Wurzel "werka- 'Werk' gehört (Kroonen 2013: 6oo). Für lat. iniquitatem benutzen die altenglischen Texte meistens unrihtwisnisse 'Ungerechtigkeit' und nur zweimal unriht 'id..' Notker hat eine etwas freiere Übersetzung, aber Vind. und Windb. benutzen verwandte Wörter.

5. Ps. 11,2: SAlvUm me FAC DOMINE QUONIAM DEFECIT SANCTUS 'rette mich, Herr, denn der Heilige ging weg' erscheint als nasei mik frauja unte fairlag weihs aippau airkns in Bologna, Bl. Ir, Z. gf. Am Anfang wird wieder das Verb nasjan 'retten' gebraucht, vgl. Nr. 2. Die westgermanischen Texte wählen dafür pgm. *haldan- bzw. Kombinationen mit der Wurzel pgm. *hail-. Got. fairlag ist ein Hapax; es handelt sich wohl um eine Lehnübersetzung von gr. ek-leípô, das in Luk. 16,9 mit ufligan wiedergegeben wird. Das Adjektiv airkns 'heilig, fromm' wird hier offenbar als Variante von weihs 'heilig' gesehen. Das gotische Wort ist nur hier belegt, denn die Lesung airkn[i]s in I Tim. 3,3 (Ambros. B) ist nicht korrekt (so Falluomini 2017: 291 mit Verweis auf Snædal 2013). Wohl findet sich noch das Adjektiv unairkns 'unheilig' (II Tim. 3,2: unairknai (B) und unairknans (A) und unairknaim (AB) in I Tim. 1,8$)$ und das Substantiv airknipa 'Echtheit' (II Kor. 8,8, sowohl in A als B). Die anderen germanischen Sprachen kennen die Wurzel zwar auch, aber sie ist dort relativ selten. Im Althochdeutschen findet sich erkan 'hervorragend, ehrwürdig, fest, bestimmt' (EWA II,1140-42) in den Glossen, im 'Isidor' und bei Notker und vereinzelt auch als Übersetzung von sanctus im Glossar Ra des Abrogans (Gll. I,12,28). ${ }^{13}$ Sonst erscheint es als erstes Element in Personennamen wie Erkanbald. Im Altenglischen und vereinzelt im Altsächsischen kommt es als Element in Personennamen vor. Ob ae. eorcnan-, eorcan, eorclanstan 'Edelstein' und an. jarknasteinn 'Edelstein' etymologisch damit verwandt sind, ist umstritten (EWA II,1141); es könnte sich hier auch um ein Lehnwort handeln. Da das Wort nur im Althochdeutschen und Gotischen und spurenweise im Altenglischen und Altsächsischen vorkommt, dürfte es sich hier um eine alte Wurzel handeln, die sich im Nordwestgermanischen auf Dauer nicht hat halten können. Die Tatsache, dass es im Gotischen als Variante von weihs 'heilig' erscheint,

13 Ob dies als Beleg für die Bedeutung 'heilig' gelten kann, ist fraglich, da alle Parallelhandschriften ahd. hèr 'erhaben' haben, vgl. Baesecke 1930: 99. 
könnte darauf hindeuten, dass das Wort auch in dieser Sprache nicht mehr so üblich war. Die anderen germanischen Sprachen bevorzugen die Wurzel pgm. *hail-, namentlich in der Form des Adjektivs pgm. *hailag- für 'sanctus': Heil mich mache herre want nicht ist der heilige (Vind.) und Heilen mich tuo herro wande zegangen ist der heilige (Windb.). Auch das Altniederländische hat in den Psalmen heilig für 'sanctus', vgl. Quak 1975: 88. Notker hat in diesem Psalmvers: Duô mih trúhten geháltenen in énero werlte. uuanda hiêr sanctus zegángen ist. Wulfila scheint dieses Adjektiv zu meiden, obwohl die Runeninschrift von Pietroassa anzudeuten scheint, dass das Wort auch im Gotischen existierte: wih [h]ailag. Im Altenglischen ist man sich auch wieder ziemlich einig:

halne me doo dryhten for ðon asprong se halga (Vesp.); halne me gedó dryhten forđon asprong se halga (Junius); halne [ ] teorode halig (Regius); halne me gedo drihtyn forpon asprong se halga. (Cambridge); gehcel me [ ] forðan pe ateorode se halga. (Lambeth); halne me do drihten forðon geswiðrað haligne (Vitellius); halne me do drihten forðon geswiprap halig (Arundel); gehcel me do drihten forðon ateorode halig (Stowe); halne [ ] teorode halig (Tiberius); halne me do [ ] forðan ateorod hali (Salisbury); hcelne me do drihten forðon pe ateorode se hceli (Canterbury); Gehcel me, Drihten, for pam haligdom is nu on pisum tidum fullneah asprungen (Paris).

Nur die etwas freiere Übersetzung in der Pariser Handschrift weicht hier ebenso wie in den meisten anderen zitierten Stellen ab. Für lat. defecit werden in den westgermanischen Sprachen unterschiedliche Wiedergaben gewählt: bei Notker und in Windb. ze(r)gehen 'vergehen' und im Altenglischen aspringan 'versagen, aufhören' (вт 54), ateorian 'versagen, aufhören' (B 57 ) und geswidrian 'schwach werden, vernichten' (вт 418). Abgesehen davon weisen die meisten Texte mehr oder weniger denselben Text auf.

6. Ps. 11,8: tu Domine Servabis nos et Custodies nos a GeneraTIONE HAC ET IN ÆTERNUM hat ein Äquivalent in: [akei] pu frauja bairgais unsis jah gawitais unsis faura kunja pamma du aiwa '[aber] Du, O Herr, mögest uns bewahren und Du wirst uns beschützen vor diesem Geschlecht bis in Ewigkeit' in Bologna, Bl. Ir, Z. 5-7. Falluomini kommentiert hier, dass in der Kombination du aiwa im Gotischen die Konjunktion jah 'et' fehlt, die in dieser Stelle normalerweise in den griechischen und 
lateinischen Versionen des Psalters stehen (2014: 292). Auch in den altenglischen Texten fehlt sie. ${ }^{14}$ Got. bairgan ist eine der möglichen Übersetzungen von gr. phulássô, wie in Joh. 12,25. Auch in den anderen germanischen Sprachen ist das Verb in dieser Bedeutung belegt, vgl. EWA I,554-56. Allerdings verwenden die westgermanischen Übersetzungen an dieser Stelle pgm. *haldan- u.ä. Got. gawitan 'schützen' erscheint auch im altenglischen witian 'hüten, bewahren' und ahd. wizzên 'id.. Die ursprüngliche Bedeutung war wohl 'sehen', vgl. Kroonen 2013: 589. In den anderen germanischen Sprachen werden Formen von pgm. * (ga)haldan 'schützen, bewachen' in dieser Stelle bevorzugt. Notker schreibt: $D u$ truhten haltest unsich. hínnan unz in êuua und hat somit beide Verben in einem Wort zusammengefasst. Die beiden frühneuhochdeutschen Psalter haben in diesem Vers behalten und behuoten. In den altenglischen Psaltern findet man:

Ju dryhten aldes usic 7 gehaldes usic from cneorisse Jisse in ecnisse (Vesp.); pu dryhten healdes us 7 gehealdes us from cneorisse pisse on ecnesse (Junius);

$\partial u[$ ] gehealdst us 7 beweardast us fram cnerisse Jisre on ecnisse (Regius); pu drihtyn healdyst us 7 gehealdyst us from cynne pisse on ecnysse (Cambridge);

pu eala ${ }^{15} \mathrm{dr}$ [ihten] gehealtst us 7 gescildst us fram cneorisse t moegðe pysre symle oððe cefre t. on ecnisse (Lambeth);

pudrihtengehealdestus 7 pugehealdest [ ]s [ ] ]sseonecnesse(Vitellius);16 pu drihten gehealdest us 7 pu gehealdest us fram cneorisse on ecnesse (Arundel);

pudrihten gehealdst us 7 bewéardast us fram cynryne oð onécnysse (Stowe); pu [ ] gehealdst us 7 beweardast us fram cneorisse pysre on ecnisse (Tiberius);

pu hlaford gehealdest us 7 beweardest us fram cneornisse pysse on ecnesse (Salisbury);

pu drihten geheceldest us 7 beweardest us fram cnyorisse Jisre on ecnesse (Canterbury);

Pu, Drihten, gehcelst us and gefreoðast fram heora yfle on ecnesse. (Paris).

14 Im lateinischen Text der Vulgata fehlt et in einigen Handschriften, vgl. Vulgata 1969: 780. Auch in den Übertragungen Vind. und Windb. fehlt dieses Wort ebenso wie bei Notker.

15 eala 'o' (вт 227) wurde hier wie häufiger in dieser Handschrift - s. Ps. 21,1 (unten Nr. 9) hinzugefügt, vgl. Pulsiano 2001: 122.

16 Teile des Textes fehlen jetzt in der Handschrift. 
Es sieht so aus, dass alle altenglischen Texte lat. servabis mit Formen von (ge-)healdan 'bewahren, beschützen' wiedergeben. Auch im Gotischen existiert dieses Verb, aber es scheint hier eher eine konkrete Bedeutung zu haben: 'halten, füttern, grasen lassen'. Bei custodies scheinen einige Texte vielleicht bewusst mit anderen Verben zu variieren, was auch für die kontinentalen Texte gelten könnte, vgl. Leningrad: Du here salt ons behouden ende bewachten. Im Altenglischen wird lat. generatio mit variierenden Übersetzungen wiedergegeben: cneoris, cneornis (вТ 162), cyn (вт 183) und cynren (вт 168).

7. Ps. 11,9: IN CIRCUITU IMPII AMBULANT. Bisunjane unsibjai gaggand 'die Gottlosen gehen herum' in Bologna, Ir, Z. 4f. Got. bisunjane Adv. 'rund herum' ist ein spezifisch gotisches Wort (Lehmann 1986: 73). Die Wiedergabe von impius 'Ungläubiger, Sünder'17 mit unsibjis kommt im gotischen Text der Psalmen noch einmal vor, s. oben Nr. 4. Das Gotische weicht hier von den anderen germanischen Sprachen ab. Dort erscheint zwar auch eine Parallele zu unsibjis etwa ahd. unsippi 'nicht verwandt' vgl. ae. ungesibb 'feindlich' (вт 1113) -, aber die Bedeutung 'Ungläubiger, Sünder' fehlt hier. Die Wurzel *sibbj- bedeutet in den anderen Sprachen nur 'Verwandtschaft', wie übrigens auch got. sibja. ${ }^{18}$ Lehmann sieht darin PIE * $s(w) e-b h o-$ 'of one's own kind' (S. 300). Möglicherweise werden die Gläubigen hier als 'verwandt' angedeutet, vgl. Kroonen 2013: 429. Dass unsibjis im Gotischen 'Gottloser' bedeutet geht auch aus einer Glossierung hervor: unsibjaim in Tim. 1,9 wird in Handschrift A mit afgudaim 'gottlos' glossiert. Im Westgermanischen werden andere Wörter verwendet: Notker hat in dieser Stelle Diê árgen gânt umbe und die frühmittelhochdeutschen Texte wählen die ubelen (Vind.) und die unguoten (Windb.). Im Altenglischen erscheinen folgende Übersetzungen:

in ymbhwyrfte ða arleasan gongað (Vesp.);

in ymbhwyrfte pa arlesan gongad (Junius);

in trundulnisse arlease gangað (Regius);

on ymbhwyrfte para arleasra hi gondgað (Cambridge):;9

on embhwyrfte l. eall abutan pa arleasan gað oððe farap (Lambeth);

onymbgange paa[ ] ]að(Vitellius);

on ymbgange pa arleasan gangep (Arundel);

17 Notker gebraucht hier Dî árgen 'die Schlimmen'.

18 Vgl. Kroonen 2013: 429.

19 Der Übersetzer hat hier 'im Kreise der Gottlosen gehen sie umher' verstanden, vgl. Wildhagen 1964: 22, Anm.13. 
on ymbhwyrfte arlease gangað (Stowe);

on trundelnesse arleasce gangað (Tiberius);

on ymbehwyrfte arlease gangað (Salisbury);

On ymbhwyrfte pce cerlecesce geengce (Canterburry);

Deah pa unrihtwisan us utan began on celce healfe (Paris).

Hier wird also für lat. impii im allgemeinen das Adjektiv ārleas 'schlecht, gottlos' (вт 50) bevorzugt. Nur die Version des Pariser Prosatextes wählt unrihtwis 'ungerecht, schlecht' (вт 1125), aber sie hat eine viel freiere Übersetzung des ganzen Verses. Nur Regius und Tiberius haben trundulnisse für circuitus, vgl. Wiesenekker 1992: 148, der darin eine Lehnübersetzung sieht.

8. Ps. 13,1: DIXIT INSIPIENS IN CORDE SUO NON EST DEUS 'der Unkluge sprach in seinem Herzen: nicht ist Gott'. Dieser Vers erscheint als qap unfrops in hairtin seinamma. nist gup in Bologna, Bl. IIr, Z. 18f. Notker hat hier Der únuuizzigo 'der Unkluge'. Andere Texte haben Wörter mit ähnlicher Bedeutung: der tumbe (Vind.) und der unwise (Windb.). Letzteres Wort erscheint auch im mittelniederländischen Leningrader Psalter und in den meisten altenglischen Texte. Die gotische Bildung unfrops 'unklug' hat Parallelen in fast allen germanischen Sprachen. Es handelt sich um die o-Stufe eines Adjektivs zu einem starken Verb, das in got. frapjan 'denken, erkennen, verstehen' erhalten ist, vgl. ahd. frad 'tüchtig', fradi 'Wirksamkeit', fradlicho 'eifrig', frado 'wirksam' und fredigo (?) und die Ableitungen gifrad 'wirksam' und gifradî Wirksamkeit', die alle selten sind; sie erscheinen nur ein- oder zweimal in den Glossen, cf. Kroonen 2013: 156 s. auch EWA III,506-07. Im Altenglischen findet sich:

cweð se unwise in his heortan nis god (Vesp.);

[ ] se unwisa in heortan his nis god (Junius);

cwceð se unwisa on heortan his [ ] (Regius);

cwceð se unwisa in heortan his nys god (Cambridge);

scede t. cwceð se unsnotera t. se unwita on heortan his nys god (Lambeth);

cwceð unwis in heortan his ne is god (Vitellius);

cwcep unwis on heortan his na is god (Arundel);

scede se unwisa on heortan his nis god (Stowe);

cwced unwis in heortan his na is god (Tiberius);

cwced unwisse on heortan heora na is god (Salisbury);

cwced se unwise t unsnotrce on herte his nis god (Canterbury);

Se unrihtwisa cwyð on his mode: "Nis nan God pe pis wite oððe wrcece." (Paris). 
Interessant ist auch, dass zwei Texte statt cwedan die Variante secgan zu bevorzugen scheinen. Nur Lambeth benutzt andere Wiedergaben für insipiens; sonst sind sich die altenglischen Psalter sehr einig mit Ausnahme des Pariser Textes, der ja öfters freier übersetzt.

9. Ps. 21,2: DeUS, DeUS MeUS, ResPiCe [IN $]^{20}$ ME: QUARE ME DERELIQUisTi? 'Gott, mein Gott, siehe auf mich. Warum hast du mich verlassen'. Im Gotischen erscheint dafür gup meins, gup meins, duhve mis bilaist? in Matt. 27,46. Das hier benutzte Verb ist got. bileipan 'weggehen'. Die westgermanischen Texte scheinen das starke Verb farlâtan 'verlassen' zu bevorzugen, z.B. Ziu ferliêze du mih? (Notker Ps. 21,2), warumbe du mich uerlieze (Vind.) bzw. warumbe mih has du uerlazzen (Windb.) vgl. ne farlattu mi 'ne derelinquas me' in WPs. (Ps. 70,9). Das Gotische kennt zwar dasselbe Verb, aber hier bedeutet es 'verzeihen, vergeben'. Die altenglischen Texte haben dieses Verb auch einheitlich:

god god min geloca in me for hon me forleorte Ju feor from hcelu (Vesp.);

[ ] god min loca on me forhwon me pu forlete feor from hcelo minre (Junius);

[ ] beseoh on me hwy [ ] Ju forlete feor fram hcelo minre (Regius);

o eala god min geloca on me forhwon me forlete pu feorr from hcele minre (Cambridge);

eala pu god god min beseoh on me forhwi me forlete pu feor fram hcele. (Lambeth);

god god min beseoh t beheald ( $t$ geloca on) me hwi me pu forlete feor fram hcele minre (Vitellius);

god god min geloca on me forhwon forlete pu me feor fram hcelo minre (Arundel);

god god min beseoh me forhwý me Ju forlete feor fram hcelo minre (Stowe); [ ] ]beseoh [ ] hwi [ ] puforlete feor fram hcelo minre (Tiberius); [ ] godmin besceawa me hwipumeforlete feorfram hcele minre.(Salisbury); God god min loce on me forwcen forlete pu me fyor fram hele minra (Canterbury);

Drihten, Drihten, min God, beseoh to me; hwi forlete pu me swa feor minre hcelo? (Paris);

10. Ps. 21,9: SPERAVIT IN DOMINO ERIPIAT EUM SALVUM FACIAT EUM QUONIAM VULT EUM. 'Er hoffte auf den Herrn: möge er ihn retten, wenn er ihn will'. Dieser Vers wird zum Teil als: trauaida du guda; lausjadau nu ina, jabai wili ina in Matth. 27,43 zitiert. Das Verb trauan 'glauben, hoffen' ist in allen germanischen Sprachen überliefert, vgl. anl. trûon 'sperare', 
und wird in dieser Stelle im Westgermanischen auch ab und zu benutzt: er getruote an got der ledige in er behalten tů in want er wil in (Vind.). Auch das schwache Verb lausjan 'erlösen, retten' kommt häufig vor. Notker hat hier: Ze góte fersáh er sih. der chóme unde lose in. Dasselbe Verb erscheint auch als Variante in einigen altenglischen Texten, obwohl dort (ge)neran vorherrscht. Im Altenglischen findet man:

gehyhteð in dryhten genere $\mathrm{x}$ hine halne doe hine for ðon he wile hine (Vesp.); gehyhted on dryhtne genered hine halne ded hine fordon he wile hine (Junius);

he gehyhte on dryhtne he alysep hine halne gedep [ ] he wile [ ] (Regius); gehihte on drihtne generyð hyne halne dóó hine forpon he wyle hyne (Cambridge);

he gehihte on drihtne he genereð t. he generie hine he gehcele hine forðan pe he wile ine. (Lambeth);

he gehihte [ ]ndrihtn [ ] he alysed (tgenerede) hine halne geded (tgedyde) hi:[ ] forpon pe he wile (t wolde) hine (Vitellius);

gehihton on drihtne generede hine halne gedide hine forpon pe he wolde (Arundel);

hig hopudon on drihtne he generað hine halne he gedep hine forðon he wile ine (Stowe);

he gehyhte on drihten he aleseð hine halne he gedep hine forðon he wile (Tiberius);

he hihte on drihten he alysde hine hal dep hine forðan wyle hine. (Salisbury); he gehyhte thopade on drihten he hine genercep t alysep halne he gedep hine fordan he wile hine (Canterbury);

He hopode to Drihtne, alyse he hine nu he gealp pret he hine lufode (Paris).

Im Altenglischen wird in den älteren Psaltern meistens (ge)hyhtan 'hoffen' (вт 404) für sperare gebraucht, obwohl de Pariser Text das Verb hopon hat, wie auch Stowe und Canterbury. Für lat. eripere erscheinen sowohl generian 'retten' (BT 421) als alýsan 'erlösen' (B 35 ).

11. Ps. 23,1: Domini est terra et plenitudo eius 'des Herren ist die Erde und was sie erfüllt'. Dieser Vers wird zitiert als: fraujins ist auk airpa jah fullo izos in I Kor. 10,26. Das lässt sich mit Notker vergleichen: Trúhtenis ist diû erda. unde íro fulli. Auch die frühmittelhochdeutschen Psalter haben ähnliche Übersetzungen: Gotes ist diu erde un[de] erfillunge ire (Vind.) und Des herren ist diu erda und uolle ire (Windb.). Im Altenglischen erscheinen: 
dryht [nes] is earðe 7 fylnis his (Vesp.);

[ ] is eorde 7 fylnes his (Junius);

drihtnes is eorðe 7 gefyllednis hire (Regius);

drihtnys is eorðe 7 gefyllydnys his (Cambridge);

drihtnes is eorðe 7 fulnysse l. gefyllednes hyre (Lambeth);

drih[tnes] is teorðan 7 gefylled(nes tfylnes) hyre (Vitellius);

drih[tnes] is eorpe 7 fylnes his (Arundel);

drihtnes is eorðe 7 gefyllednisse hire (Stowe);

drihtnes is eorðe 7 gefyllednis hire (Tiberius);

[ ] is eorpe 7 gefyllednes his (Salisbury);

Drihtnes is sio eorpe 7 gefelledness hire (Canterbury);

Drihtnes ys eorðe and eall paet heo mid gefyld is (Paris).

Hier haben das Gotische und die westgermanischen Sprachen mehr oder weniger dieselben Übertragungen. Nur dass im Westgermanischen eher *druhtin, hèrro oder god statt *frô- gebraucht wird.

12. Ps. 32,15: QUI FINXIT SINGILLATIM CORDA EORUM. QUI INTELLEGIT SUPER OMNIA OPERA EORUM. 'Er hat sie einzeln erschaffen die Herzen von allen; der über allem ihre Werke versteht'. Der Psalmvers erscheint im Gotischen als: saei daig ainhvarjammeh hairtona ize. Saei frapjip in alla waurstwa ize (Bologna, Bl. Iiv, Z.13f.). Got. daig gehört zum starken Verb digan 'kneten, formen', das etymologisch mit lat. fingere verwandt ist, vgl. Kroonen 2013: 95. Das griechische substantivierte Aoristpartizip ist wie oft durch einen Relativsatz mit dem Präteritum wiedergegeben. Das Verb erscheint im Gotischen sonst nur als Partizip digandin (Röm. 9,20), kasa digana 'irdene Gefäße' (II Tim. 2,20) und gadigan in I Tim. 2,13 (A und B). Obwohl die Wurzel auch in den anderen germanischen Sprachen vorkommt, ist das Verb nur im Gotischen in dieser ursprünglichen Bedeutung erhalten. Die anderen germanischen Sprachen gebrauchen für lat. fingere Verben mit Bedeutungen wie 'schöpfen' oder 'bilden': Notker: Der ein-luzlîcho íro herzen scuôf, oder anl. bilithon in gibilithoda 'finxit' im selben Psalm, vgl. auch der erdahte besunter div herce ire der da verstet elliv werch ire (Vind.) und Der der gebilidet hat sunterliche. einlichen herce ire derder entstet. uernimit elliu werh ire (Windb.). In den altsächsischen 'Lubliner Psalmen' fehlt im Fragment gerade das Verb. Sowohl Notker wie die altenglische Übersetzung im Pariser Psalter übertragen etwas freier mit pgm. "skapjan- 'schöpfen', während das Gotische und der altniederländische Text die ursprüngliche Bedeutung ‘bilden, formen' festhalten. Die englischen Psalter haben: 
se gehiewade wrixendlice heortan heara se ongiteð in allwerc hiera (Vesp.); se gehiwode wrixendlice heortan hira se ongited on eall weorc hira (Junius); se hiwode todceledlice heortan heora se angyt on ealle weorc heora (Regius); se gehiwude wrixiyndlice heorte hyra se ongytyð on ealle weorc heora. (Cambridge);

se pe gehywode synderlice heortan heora se pe ongytt ealle worc heora. (Lambeth);

pe hiwode todcelendlice heortan heora se ongyt ealle weorc heora. (Vitellius); gehiwode wrixendlice heorte heora se pe ongitep ealle weorc heora (Arundel);

se hywode todceledlice heortan heora se ongyt ealle weorc heora (Stowe); se hiwode todceledlice heorta heora se angyt ealle weorc heora (Tiberius); se hiwode todceledlice heorta heora se angyt ealle weorc hera (Salisbury); se hiwode Sienderlice hiorce heortcen pce angetcep on ealle hiorce weorc (Canterbury);

for pam he gesceop heora heortan, celces synderlice, and he ongit heora ealra weorc (Paris).

Das Verb gehiwian bedeutet 'formen, bilden' (BT 401). Got. ainhvarjammeh 'katá mónas' ist eine ungewöhnliche Wiedergabe des griechischen Ausdrucks, vgl. Luk. 9,18 und Mark. 4,10: sundro. Man vergleiche aber Rom. 12,5 ainhvarjizuh 'kath'eís'. Die altenglischen Texte benutzen hier wrixendlice 'um und um, eins nach dem anderen' (вт 1276), bzw. todceledlice 'einzeln, nicht in Verbindung' (вт 996). Nur Canterbury benutzt das mit den kontinentalgermanischen Formen verwandten synderlice mit derselben Bedeutung (вт 964). In den Lubliner Psalmen fehlt gerade die Übersetzung von lat. singillatim. Das starke Verb frapjan 'denken, verstehen' ist spezifisch gotisch. Die Wurzel erscheint allerdings auch in anderen germanischen Sprachen (vgl. Nr. 8) und es ist möglich, dass das Gotische dieses Verb und die Ableitungen davon besser behalten hat. Die Wurzel scheint ja im Gotischen noch ziemlich lebendig zu sein. In den 'Lubliner Psalmen' findet sich hier im letzten Teil des Verses: thiethe forstaid alle uuerk ira. Das Altenglische scheint das Verb ongitan 'wahrnehmen, wissen, verstehen' (вт 753) zu bevorzugen, das auf dem Kontinent nur im Althochdeutschen einmal erscheint: intgezzan 'erkennen' im Abrogans (EWA IV,2O2).

13. Ps. 35,2: Dixit iniUstus Ut Delinquat in SEMEt ipso 'sagte der Gottlose, damit er sündige gegen sich selbst'. Der Vers wird zitiert als: qap unsibjis ei frawaurkjai in sis silbin in Bologna, IIr, Z. 20. Falluomini erwähnt, dass got. ei frawaurkjai einem lat. ut delinquat bzw. delinquit entspricht (2014: 293). Sowohl im Gotischen wie im Lateinischen sei die 
Wiedergabe mit einem substantivierten Infinitiv, wie im Griechischen, unmöglich. Notker hat hier: Der unrehto. chît der propheta. geêinota sih sáment ímo sélbemo. daz er míssetuôe, vgl. damit ähnlich: Sprach der unrehte daz nieht ${ }^{21}$ missetu an ime selbem (Vind.) und Sprah der unrehte daz er missetuo in ime selbeme (Windb.). Im Altenglischen findet man:

cweð se unrehtwisa ðrette [ ] in him seolfum (Vesp.); cwced se unryhtwisa prette agylte on him selfum (Junius); cwce se unrihtwisa pret he agylte on selfum him (Regius); cwceð se unrihtwisa pcette he forlcete ${ }^{22}$ on hym sylfum (Cambridge); scede t. cwce se unrehtwisa pret he agylte on him sylfum (Lambeth); scede tcwce se unrihtwisa pret he agylte on sylfum him (Vitellius); cwcep se unrihtwisa pret pe agilde on hym sylfum (Arundel); cwcep se unrihtwisa pret he agylte on hym sylfum (Stowe); cwceð se unrihtwisa [ ] he agylte on selfum him (Tiberius); cwce pe unrihtwisa pret he agulde on sylfum him (Salisbury); cwceð se unrihtwise pet he forlet t agylte on him Selfum (Canterbury); Se unrehtwisa cwyp on his mode paet he wylle syngian (Paris).

Die altenglischen Texte mit Ausnahme von Paris haben für delinquat meistens Formen von agiltan 'sündigen' (вт 29), aber Arundel und Salisbury scheinen Formen von agildan 'vergelten' zu bieten. Für Arundel gilt dann wohl, was der Herausgeber Oess S. 6 schreibt, nämlich dass sich hier viele Fehler im Text finden. Auch der Schreiber von Salisbury machte viele Fehler (Sisam \& Sisam 1959: §34ff.) und als solcher ist hier wohl auch die Form agulde in diesem Vers aufzufassen.

14. Ps. 37,22: NON ${ }^{23}$ DERELINQUAS ME DOMINE MEUS NE DisCESSERIS A $\mathrm{ME}^{24}$ 'verlass mich nicht Herr, mein Gott, entferne dich nicht von mir'. Der Vers erscheint als [bi hvana qap praufetus]. gup meins ni fairjais puk af mis. [jah anpara managa.] '[über den der Prophet sagte:] mein Gott du mögest dich nicht von mir entfernen [und vieles andere]' in Bologna Bl. IIv, Z. 1of.: Got. fairjais ist ein Hapax. Das griechische Verb wird sonst mit afgaggan und afstandan wiedergegeben. Das Verb erscheint auch in anderen germanischen Texten in derselben Bedeutung, etwa in den WPs. firron 'elongere' in Ps. 54,8 and 72,27. Notker hat hier: Ne-ferlazest dû mih

\footnotetext{
21 Im lateinischen Text steht hier non.

22 Variante: derelinquat. Die Verben delinquere und derelinquere werden in den Texten oft verwechselt, vgl. die Übertragung in Canterbury.

23 Variante: $n e$ in einigen lateinischen Handschriften und auch in der Vorlage einiger altenglischen Übertragungen..

24 vgl. die Variante im Psalterium iuxta Hebraeos: ne elongeris a me.
} 
trúhten Got mîner. noh dû ne-sceidest fóne mir und die beiden jüngeren Psalter: niht uerlaze mich herre got miner nieht entwiche uon mir (Vind.), Nihne uerlazzes du mih herro got min daz iht engest du uone mir (Windb.). Die altenglischen Psalter haben folgende Formen:

ne forlet ðu me dryht [en] god min ne gewit ðu from me (Vesp.);

[n] e forlcet đu me dryht [en] god min ne gewit đu from me (Junius); neforlcete ðu [ ] gewite ðu [ ] (Regius); ne forlcet pu me dryht [yn] god min ne gewit ðu fram me (Cambridge); ne forlcet ðu me eala $p u^{25}$ min god min ne gewit pu fram me (Lambeth); ne forlcet pu me driht [en] god min ne gewite pu fram me (Vitellius); ne forlcet pu me drih [ten] god min ne gewit pu fram me (Arundel); ne forlcet pu me driht [en] god min ne gewit pu fram me (Stowe); ne forlcete pu [ ] ne gewite pe pu fram me (Tiberius); na forlcet pu me [ ] god min na gewit fram me (Salisbury); Ne forlet pu me drihten min god ne gewite ðu from me (Canterbury); Ne forlcet me, Drihten, min God, ne ne gewit fram me. (Paris).

Alle westgermanischen Texte benutzen somit am Anfang des Verses das Verb *far-lâtan 'verlassen', Im zweiten Teil war die Auswahl offensichtlich größer, wenn auch die altenglischen Texte alle das Verb gewitan 'weggehen, verlassen' (в 4 469) benutzen, vgl. Nr. 4.

15. PS 40,10: ETENIM HOMO PACIS MEÆ IN QUO SPERAVI QUI EDEBAT PANES MEOS MAGNifiCAVIT SUPER ME SUBPLANTATIONEM. ${ }^{26}$ 'auch der Mensch meines Friedens, dem ich traute, der mein Brot aß, hat mich betrogen'. Der Text saei matida mip mis hlaib, ushof ana mik fairzna

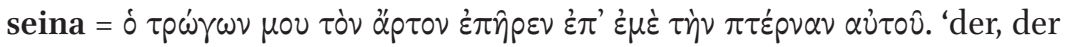
mit mir Brot aß, hat seine Ferse gegen mich erhoben' findet sich in Joh. 13,18. Got. fairzna 'Ferse' erscheint nur hier, aber ist allgemein in anderen germanischen Sprachen, vgl. anl. fersna in Ps. 55,7 und ahd. fersna (EWA III,174-76). Der Ausdruck ist hier eine genaue Übersetzung des griechischen Textes: got. us-hafjan 'aufheben' wurde mit fairzna 'Ferse' kombiniert. Die lateinische Fassung gebraucht ein anderes Wort: supplantatio 'heuchlerische Täuschung' und hier wird der Unterschied zwischen dem gotischen Text mit seiner griechischen Tradition und dem Westgermanischen mit der lateinischen Tradition wieder sichtbar. Man vergleiche Notker: der mit mir âz. der bescráncta mih uuitto. 'Derjenige,

26 In den altenglischen Psaltern findet man hier auch sperabam statt speravi und ampliavit statt magnificavit (Pulsiano 2001: 593). 
der mit mir aß, hat mich schwer betrogen', mit ahd. biscrenken 'betrügen' (Schützeichel 2006: 312). Die frühmittelhochdeutschen Psalter wählen hier andere Wörter: grozlichen tet er uber mich sine trunchenheit ${ }^{27}$ (Vind.) und der gemihlichte grozlichte uber mih die unterscuphunge 28 (Windb.). Im Altenglischen findet man in einigen Psaltern für subplantatio eine Ableitung von derselben Wurzel wie bei Notker: gescrencednes (вт 437) in Variation mit ae. bigsprec 'Betrug' (вт 100):

se ðe et hlafas mine gemonigfaldade wið me gescrencednisse (Vesp.); se de cet hlafas mine gemonigfaldode wid me gescrencednesse (Junius); se cet hlafas mine geycte ongean me bygswcenc ${ }^{29}$ (Regius); se ðe et hlafas mine gemonigfealdode wið me gescrencednesse (Cambridge); se ðe cet hlafas mine miclode ofor me hleohrcescnisse t. forsc [r] cencednysse (Lambeth); se ðe cet hlaf minne gemcersode ofer me byg [ ] $]^{30}$ swencednesse (Vitellius); se pe cet hlafas mine gemicclode ofer me gescrcencednesse (Arundel); se pe cet hláfas mine sý micelod ofer me bygspæec (Stowe); se pe cet hlafas mine [ ] bygswec (Tiberius) pa eet hlaf minne he gemyclað ofer me bigspæec (Salisbury); pce pe cet minne hloef geycte t monigfeceldode ongean me underpidnesse (Canterbury);

Die Übertragung von lat. supplantatio machte den englischen Übersetzern offenbar Schwierigkeiten, denn es findet sich hier eine reiche Variation. Dabei bietet hleohrescnisse in Lambeth Schwierigkeiten bei der Interpretation, vgl. BT 543 und Suppl. 551. Wie öfter weicht der Pariser Text mit seiner freieren Übersetzung von den anderen altenglischen Texten ab: ge furðon pa sprcecon pret ylce mid him, pe ic betst truwode, and pa pe cer ceton and druncon mid me (O'Neill 2016: 146).

16. Ps. 61,8: In Deo SAlutare meum et Gloria mea. Gotisch: in guda naseins meina jah wulpus meins (Bologna, Bl. Iv, Z. 17-18) 'in Gott (ist) meine Rettung und meine Glorie'. Für 'Rettung' erscheint hier dasselbe

27 Eine Deutung dieser sonderbaren Übersetzung ist schwierig, aber vermutlich hat Kirchert recht, wenn er annimmt, dass es sich um einen Schreibfehler für trugenheit 'Betrug' handelt (1979: 183-84).

28 Vgl. mhd. under-schupfen 'mit List verdrängen' (Lexer II,180o).

29 Roeder macht in einer Fussnote darauf aufmerksam, dass dieses Wort und bygswec im Tiberius Psalter zum Verb biswîcan 'betrügen' gehören können und somit keine Fehler für bisprec (Salisbury und Stowe) zu sein brauchen, vgl. anl. besuîk 'supplanta' (Ps. 16,13) in den WPs.

Ein Teil des Wortes fehlt. Man lese wohl bygspcec oder ähnliches. 
Wort wie in Nr. 1. Das Wort wulpus 'Glorie' erscheint in dieser Bedeutung nur im Gotischen und vielleicht auch im Namen WulpupewaR in einer Runeninschrift aus Thorsbjerg, s. auch den Namen des Gottes Ullr im Altnordischen. Das Wort dürfte jedoch mit ae. wuldor 'Glorie' in den unten zitierten Psalmtexten verwandt sein, cf. Kroonen 2013: 599. ${ }^{31}$ Dieser Vers kann man vergleichen mit An gode salda min in guolicheide min (WPs.) und Notker: An Góte ist mîn heîlhafti. unde min guôllichi. Diese beiden Texte benutzen die verwandten Wörter guolicheide und guôllichi für 'Glorie'. Die beiden frühmittelhochdeutschen Psalter benutzen hier ere. Im Altenglischen erscheinen:

in gode ðœеm halwyndan minum 7 wuldur min (Vesp.);

on gode prem halwendan minum 7 wuldor min (Junius);

on gode hrelo min 7 wuldur [ ] (Regius);

on gode pam halwendan min 7 wuldor min (Cambridge);

on gode min halwenda 7 min wulder (Lambeth);

on gode hoelo min 7 wuldor min (Vitellius);

on gode hcelo min 7 wundor min (Arundel);

on dcege $e^{32}$ hrelo mine 7 wuldor min (Stowe);

on gode haelo min [ ] wuldor min (Tiberius);

on gode hoele min 7 wuldor min (Salisbury);

On godce helo min 7 wuldor min (Canterbury).

In einigen Psaltern steht in diesem Vers im lateinischen Text salutari meo 'meinem Retter' und das erklärt die Variation zwischen hâlwenda 'Heiland' (BT 506) und hcel(u) 'Rettung'.

17. Ps 67,19: Ascendisti in Altum CePisti CAPTivitatem ACCEPISTi DonA in Hominibus: 'du bis aufgefahren in die Höhe, du hast Gefangene gefangen, du hast Gaben empfangen unter den Menschen'. Dieser Vers wird zitiert in Eph. 4,8: in pizei qipip: ussteigands in hauhipa ushanp hunp jah atuhgaf gibos mannam. Im Altniederländischen erscheint dieser Vers als: Upstigi an hoi, nami hafta, antfiengi geua an mannon (WPs.) Das Eigentümliche ist, dass Eph. 4,8 über 'Geschenke geben' spricht und der Psalmvers über 'empfangen'. Der gotische Text benutzt in diesem Vers für den zweiten Teil des Verses us-himpan 'gefangen nehmen', und das Substantiv hunps 'Fang, Beute'. Auch hier hat das Gotische

31 Auch der Pariser Text kennt dieses Wort: On Gode standeð min gearu hcelu and wuldor min and wyn micel. (O'Neill 2016: 224). 
archaische Formen, denn diese Wurzel hat nur wenige Verwandte in den anderen germanischen Sprachen. Im Althochdeutschen findet man nur hunda 'Beute' in einer einzigen bairischen Glosse (EWA IV,1219) und im Altenglischen hû̃(e) 'Beute' (вт 569). Das Verb erscheint noch im Altnordischen wie etwa in aschw. hinna (Kroonen 2013: 227). Die figura etymologica ushanp hunp, eine Übersetzung von gr. ทे $\chi \mu \alpha \lambda \omega \dot{\omega} \tau \varepsilon v \sigma \varepsilon v$ $\alpha i \chi \mu \alpha \lambda \omega \sigma i \alpha \nu$, wo Wörter mit derselben etymologischen Herkunft in derselben Stelle benutzt werden, findet man nicht nur im Gotischen, vgl. z.B Notker: Dû CHRISTE stîge ze hímele. déro ménniscon éllende geéllendôtôst dû. dû irsluôge den tôd. mísselicha géba inphiênge dû án ín, mit dem Substantiv elilenti 'Verbannung, Gefangenschaft' (Schützeichel 2006: 9o) und dem Verb geellendôn 'gefangen nehmen' (ebd.: 91). Auch Vind. scheint so etwas zu haben: uffurre du in die hohe uienge die uanchnusse name du grebe in den liuten. In einigen altenglischen Psaltern lassen sich möglicherweise davon Spuren finden:

stigende ${ }^{33}$ in heanisse gehefte loedde heftned salde gefa monnum (Vesp.); stigende on heanesse gehcefte loedde hreftnede sealde gefe monnum (Junius);

astigende on heanisse gehceftne ${ }^{34}$ he loedde hoeftned he sealde selena mannum (Regius);

stigynde on heanysse Jeowe gelcedde on haeftnyd sealde gife mannum (Cambridge);

pu astige on heanysse pu gegripe hreftnunge pu gename sylena on mannum (Lambeth);

pu astige on heannesse [ ] haeftned pu onfenge sylena on mannum (Vitellius);

pu astige on heannessum [ ] haeftnyde pu onfencge gifu on mannum (Arundel);

upastigen on heannysse pu onfenge hreftnyd pu onfenge selena on mannum (Stowe);

pu astige on heannesse [ ] hreftnyd [ ] selena on mannum (Tiberius); astigende on heahnesse fenge gehreftnede underfenge gyue on mannan (Salisbury);

astigende on heahnesse gehceftne he ledde haftned he gaf giue monnum (Canterbury).

33 Dieser Psalter und einige andere haben hier die Variante ascendens, welche das Präsenspartizip erklärt.

34 Roeder nimmt an, dass man dafür gehcefte lesen soll. 
Auch hier findet man den Gegensatz 'schenken' gegenüber 'empfangen' im zweiten Teil des Verses. Es liegt wohl ein Unterschied zwischen dem Psalterium Romanum und dem Psalterium Gallicanum vor. Im ersteren findet sich dedit statt accepisti wie auch in Eph. 4,8. Die Übersetzung im Pariser Psalter ist weit freier, aber enthält im Grunde dieselben Vokabeln: on heanesse astah, haeft-ned lcedde / pa on haeft-nede hwile micele / lange lifdon, and wces lac-geofa / ofer middan-geard manna bearnum (O'Neill 2016: 246).

18. Ps. 68,5: Multiplicati sunt super Capillos Capitis Mei, QUi ODERUNT ME GRATIS. 'die mich ohne Grund hassen, sind mehr als ich Haare auf dem Haupt habe'. Der letzte Teil dieses Verses erscheint in: [ak ei usfullnodedi waurd pata gamelido in witoda ize:] ei fijaidedun mik arwjo '[aber damit erfüllt werde das Wort, das im Gesetz geschrieben steht,] sie hassten mich ohne Grund' in Joh. 15,25. Im Altniederländischen heißt es hier: thia hatodon mi thankis, so wie auch Notker hat: Mánigeren sint danne mînes hoûbetes hârer. die mich házzent tánches. Das gotische Verb fijan 'hassen' erscheint auch in den anderen altgermanischen Sprachen: an. fjá, ae. fền, fêogan, ahd. fî̀n 'hassen' (nur im 8. und 9. Jh.) wie auch das dazu gehörende Substantiv fijands 'Feind'. Aber die normale Übersetzung für lat. odi 'hassen' sind hier jedoch Verwandte des Verbs hatôn, hatên 'hassen', vgl. auch: Gemanichualtet si sint uber die loche houbites mines. die der hazzeten mih danches (Windb.). Das Adverb arwjo 'vergeblich, ohne Grund' hat einige Verwandte in ahd. ar(a)wun, arwingun und ae. earwunga 'id.' (s. unten Paris), aber die erste althochdeutsche Form findet sich nur in einigen alamannischen Texte aus dem 9. Jahrhundert und die zweite nur bis ins 12. Jahrhundert (EWA I,311-13). Das Gotische scheint auch hier wieder die altertümlicheren Formen zu haben. Die altenglischen Texte bieten:

ða fiodun mec bi ungewyrhtum (Vesp.);

es fehlt hier ein Blatt in Junius;

pa hatedon me butan gewyrhtum (Regius);

pa ðe feodon me butan gewirhtum (Bosworth);

pa feodon me be ungewyrthtum (Cambridge);

pa pe hatedun [ ] butan geearnungum (Lambeth);

pa pe hatodon mi buton (Vitellius);

pa ðe hatodon me buton gewirhtum (Arundel);

pa ðe hatudon me buton geearnungum ${ }^{35}$ (Stowe); 
pa hatodon me buton ${ }^{36}$ (Tiberius);

pa hatodon me swype (Salisbury);

pce ðe fiogcedon thatedon me buton gewryhtum (Canterbury);

pe me earwunga ealle feogað (Paris).

Im Altenglischen findet man somit wie im Gotischen fēogan in einigen älteren Psaltern, während in den jüngeren meistens hatodon erscheint. Für lat. gratis erscheinen neben be/butan ungewyrhtum 'ohne Grund' (вт 1117) sowohl bûtan geearnungum 'sine merito, gratis' (вт 387) wie earwunga (вт 235).

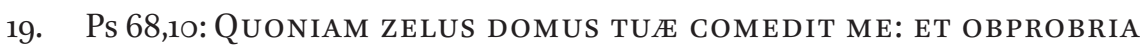
EXPRobrantium tibi, CECIDERUNT SUPER ME 'denn der Eifer deines Hauses aß mich, und die Schmähungen derer, die dich schmähten, fiellen über mich'. Der erste Teil dieses Psalmverses fehlt im Gotischen, aber in Röm. 15,3 findet man: ... pize idweitjandane puk gadrusun ana mik. Das Verb idweitjan 'schmähen' und das Substantiv idweit 'vorwerfen' haben Parallelen in anderen germanischen Sprachen, z.B. Notker benutzt im selben Vers ähnliche Formen: Vnde íteuuizza déro dir íteuuizzónton châmen an mih, vgl. auch: die itewizze dere uerwizenten dir uielen sint geuallen uf mich (Windb.) und dei itwiz itwizunter dir fielen uber mich (Vind.). Der altniederländische Text wählt hier andere Wörter: bismer lastrindero thi fielen ouer mi, wobei lastron vielleicht eine kontinentalgermanische Form ist (de Grauwe $§ 133-35$ ). ${ }^{37}$ Die Form eduuit 'opprobrium' kommt in Ps. 14,3 vor, im Rest des Textes erscheint fünfmal bismer als Übersetzung. ${ }^{38}$ Das Verb driusan 'fallen' hat Parallelen in ae. drēosan und as. driosan 'fallen' und Verwandte im Altnordischen und Althochdeutschen. Im Altsächsischen erscheint es nur einmal im 'Heliand' (Tiefenbach 2010). Vielleicht handelt es sich wieder um eine archaische Wurzel. Im Altenglischen finden sich folgende Texte:

7 edwit edwitendra ðe gefeollun ofer mec (Vesp.);

7 hospas edwitendra de feollon ofer me (Junius);

7 hospas hyspendra pe hruron [ ] (Regius);

7 hospas edwitendra 7 teelendra pe feollon ofer me (Bosworth);

36 Hier und in Vitellius soll man, entsprechend der Anmerkung bei Campbell (1974: 169), wohl buton gewyrhtum lesen, vgl. die anderen Texte.

37 vgl. EWA V,1050-52: lastar nur ahd., as. und afri., während im Altenglischen in derselben Bedeutung leahter erscheint.

38 Diese Form erscheint im Althochdeutschen nur in Glossen und bei Otfrid. Im Altsächsischen kommt es nur in der Zusammensetzung bismersprāka 'Spottrede' vor, im Altenglischen bedeutet bismer 'Spott, Beleidigung' (вт 105), vgl. EWA II,115-16. 
7 edwit edwityndra pe gefeollon ofyr me (Cambridge);

[ ] hosp hyspendra pe 7 feollun [ ] (Lambeth);

7 hospas (t edwit) hospendra t tcelendra pe [ ] me (Vitellius);

7 hospas tedwit 7 tcelendra pe feollon ofer me (Arundel);

7 hosp edwitendre pe hruron ofer me (Stowe);

7 hospas hyspendra pe hruran ofer me (Tiberius);

7 hospas hyspende pe hruran ofer me (Salisbury);

7 edwit thosp edwittendrae ðe gefiollon ofer me (Canterbury);

and me eac fela pinra edwita on gefeollon (Paris).

Für lat. opprobrium findet man hier also neben edwit auch hosp 'Vorwurf, Beleidigung' (вт 554) mit dem Verb hyspan 'vorwerfen, schmähen' (вт 585). Statt dieses letzte Verbes erscheint auch tcelan 'schmähen' (вт 969). Das Altenglische gebraucht im letzten Teil meistens feallan 'fallen', aber drei Texte haben hruron zu hreosan 'fallen' (BT 558).

20. Ps. 76,14-15: Quis deus magnus sicut Deus noster tu es Deus QUI FACIS MIRABILIA ${ }^{39}$ 'wer ist ein großer Gott wie unser Gott? Du bist der Gott der du [allein] Wunder verrichtest'. Dieser Vers wird in einem der Graffiti in Mankup (Krim) zitiert: hvas gup mikils / swe gup unsar pu / is gup waurkjands / sildaleika ainn[s] / usstop / und aiwins / us daupaim / jah in midjun[gard-] (Vinogradov \& Korobov 2014). Das Substantiv sildaleik 'Erstaunen' erscheint sonst nur noch Luk. 5,9. Man findet im Gotischen auch das Adjektiv sildaleiks 'erstaunlich, wunderbar' und die Verbe sildaleikjan 'anstaunen, bewundern' und sildaleiknan 'bewundert werden'. Die Kombination von silda und leiks ist spezifisch gotisch, wenn auch das erste Element allgemein germanisch ist, vgl. Lehmann 1986: 303 . Die anderen germanischen Sprachen bevorzugen das Substantiv wunder 'Wunder', wobei Tiberius wohl versehentlich wuldru hat. Notker: Dû bist Got. dû êino uuúnder tuôst. unde mit diû scéinest. daz ándererne-ist, vgl. du bist got du da machest wnter (Vind.). Die Variante solus in Ps. 76,15 gehört zum Psalterium Romanum, das in den älteren altenglischen Texten und in Canterbury die Basis bildet. Im Altenglischen findet man:

hwelc god micel swe swe god ur ðu earð god ðu ðe doest wunder ana (Vesp.); hwelc god micel swa swa god ure pu eart god pu de dest wundru ana (Junius);

hwylc [ ] micel swa swa [ ] pu [ ] pe destwundruana (Regius);

39 Im Psalterium Romanum und auch bei Notker und in einigen altenglischen Psaltern steht hier auch noch solus 'allein'. 
hwylc god mycyl swylc swylc god ure pu eart god pu pe dest wundru ana (Cambridge);

pu eart god pu pe wyrcst wundru ana ${ }^{40}$ (Lambeth);

hwylc god micel swaswa god ure pu eart god pu pe dydest wundra (Vitellius); hwilc god is micel swaswa god ure pu eart god pe dest wundra (Arundel); hwylc god micel swa swa god ure pu eart god se pe wyrcp wundra (Stowe); hwilc [ ] swa swa [ ] pu eart [ ] pe dydest wuldru (Tiberius); hylc god [ ] god ure ðu eart [ ] d [ ] (Salisbury); hwylc god michel swa god ure Ju eart god pe wyrchest wundor cence (Canterbury)

hwylc is mihtig god butan ure se mcera God? Pu eart ana God pe ceghwylc miht wundor gewyrca on woruld-life (Paris).

Im Großen und Ganzen haben die englischen Psalter denselben Text. Nur dass Lambeth, und Stowe das Verb dôn durch wyrcan (вт 1287) ersetzen. Offenbar ist dies eine jüngere Form, denn auch in Canterbury und in den metrischen Psalmen von Paris findet es sich an dieser Stelle.

21. Ps. 77,24: Et Pluit illis MANNA AD MANDUCANDUM, Et PANem CÆLI DEDIT EIS. 'und er ließ Manna auf sie regnen zur Speise und gab ihnen Himmelsbrot'. Der letzte Teil dieses Verses wird als: swaswe ist gamelip: hlaif us himina gaf im du matjan in Joh. 6,31) zitiert. Das Verb matjan 'essen geben' hat Parallelen in an. metja 'schlürfen (von Hunden)' und metta 'sättigen' und ae. mettian 'füttern', aber das Substantiv mats 'Essen, Speise' und andere Ableitungen von dieser Wurzel kommen in allen germanischen Sprachen vor, obwohl in diesem Psalmvers normalerweise das Verb etan 'essen' bevorzugt wird. Notker hat: Vnde régenota in manna ze ezzene. unde gáb in hímel-brôt und Windb.: Vnde regente in daz himilbrot ze ezzene und brot des himilis gab er in. ${ }^{41}$ Im Altenglischen findet man:

7 rineð him heofenlic hlaf eotan hlaf heofenes salde him (Vesp.);

7 rinde him heofonlic hlaf etan hlaf heofones sealde him (Junius);

7 rinde him heofone hlaf etan hlaf [ ] he sealde him (Regius);

7 riny him hefynlic hlaf etan hlaf hefonas sealde him (Cambridge);

7 he rinde heom pane heofonlican mete to etanne hlaf heofones 7 he sealde heom (Lambeth);

7 rinde him heofenes hlaf to etanne 7 hlaf heofenes he sealde him (Vitellius);

40 Am Rande steht solus: ana.

41 S. auch Len.: Ende hi dede hem regenen tetene manna ende dat brood des hemels gaf hi hem. 
7 rinde him heofones hlaf to etenne hlaf heofones he sealde him (Arundel); 7 rynde him mete to etanne hlaf heofonas sealde him (Stowe); 7 rinde him heofonashlaf to etenne 7 hlaf [ ] he sealde him (Tiberius); $7 \mathrm{ran}$ heom mela to etende hlaf of heofenan geaf heom (Salisbury);

7 rinde hem manne $t$ hefenlich laf to etonne hloef hefones scealde hem (Canterbury)

and hider rignan manne to mose manna cynne; sealde him heofenes hlaf hider on foldan (Paris).

Germanisch *brauda- und *hlaiba- bezeichneten vermutlich ursprünglich unterschiedliche Sorten Brot, mit und ohne Hefe. Beide Wörter kommen in allen germanischen Sprachen mit Ausnahme des Gotischen vor. ${ }^{42}$ Alle altenglischen Texte haben in diesem Vers *hlaiba- und die deutschen Texte und der niederländische Psalter *brauda-. Auffällig ist, dass nur Salisbury das Verb gefan 'geben' benutzt wie die kontinentalen Texte. Die anderen altenglischen Psalter bevorzugen sellan 'geben' (вт 861), das auf dem Kontinent eher 'übergeben, übereignen' bedeutet.

22. Ps.81,6: EgO DiXI: DiI ESTIS, ET FILII EXCElSi OMNes. 'Ich sagte: ihr seid Götter und alle seid ihr Kinder des Höchsten'. Ein Teil des Verses erscheint auf Gotisch in: niu ist gamelip in witoda izwaramma: ik qap, guda sijup? 'steht nicht geschrieben in euerem Gesetz: ich sagte, ihr seid Götter?' (Joh. 10,34). Hier wie in anderen gotischen Texten wird das Verb meljan benutzt als das normale Wort für 'schreiben'. Bei Notker findet sich: Ih chad. dii estis. filii altissimi omnes estis mit der interlinearen Glosse: Gota bind-ir des hôhesten súne bint í álle, vgl. Ih sprah gote birt ir unde chint des hohen alle (Windb.). Im Altenglischen finden sich:

ic cweð godas ge earun (Vesp.);

ic cwceđ godas ge sindon (Junius);

ic cwce godas ge syndon (Regius);

ic cwce godas ge syndon (Cambridge);

ic scede godas ge syndon (Lambeth);

ic cwceð godas ge syndon (Vitellius);

ic cwcep godas we ${ }^{43}$ syndon (Arundel);

ic cwceð godas ge syndon (Stowe);

ic cwcep godas ge syndon (Tiberius);

42 Obwohl das Vorkommen von krimgot. broe 'Brot' suggeriert, dass auch im Gotischen beide Wörter einmal existiert haben.

43 Dies ist wohl einer der vielen Fehler in Arundel, vgl. Oess 1910: $5 \mathrm{ff}$. 
ic cwepe godas ge beoð (Salisbury);

ic cwipe gode ge sindon (Canterbury);

Ic cerest cwceð: "Ge synd uppe godu" (Paris).

Es gibt keine speziellen Formen im Gotischen im Vergleich mit den westgermanischen Sprachen. Wohl fällt wieder auf, dass die englischen Texte alle fast denselben Text haben.

23. Ps. 9o,11: QUONIAM ANGelis SUis MANDABit DE TE UT CUSTODIANT te in OMNIBUS ViIS tUis. (12) In MANibus portabunt te NE FORTE OFFENDAS AD LAPIDEM PEDEM TUUM. 'denn er hat seinen Engeln geboten, damit sie dich beschützen auf allen deinen Wegen. (12) In den Händen werden sie dich tragen, damit du nicht sehr deinen Fuß am Steine stoßest'. Im Gotischen findet sich: gamelid ist auk patei aggilum seinaim anabiudip bi puk du gafastan puk (11) jah patei ana handum puk ufhaband, ei hvan ni gastagqjais bi staina fotu peinana. 'denn es wurde ja geschrieben, dass er seinen Engeln gebot mit Bezug auf dich dich zu bewahren (11) und dass sie dich in den Händen tragen, damit du nicht deinen Fuß am Stein stoßen mögest' in Luk. 4,10-11. Das Verb gafastan 'erhalten, bewahren' erscheint auch in Ps. 11,8 (Nr. 6). Im gotischen Text ist das Verb gastagqjan 'anstoßen an' interessant. Es gehört als Kausativ zu der Wurzel des starken Verbs stigqan 'stoßen'. In der Bedeutung hat dieses gotische Verb eine Parallele in an. støkkva 'springen, fliehen' und ae. stencan 'auseinanderjagen' (вт 916). Notker gebraucht in diesem Vers huoten 'behüten' bzw. ( far)stôzzan 'stoßen': Vuanda dîn fáter gebôt sinen angelis umbe dih. daz sie dîn huôten in allen dînen uuégen [...] In íro handen trágent sie dih. [...] Niê dû dînen fuôz ne-ferstôzzest an den stéin, ebenso wie Vind. und Windb. Im Altenglischen werden für das erste Verb immer healdan (vgl. Nr. 6) und für das zweite meistens Formen von spornan 'mit dem Fuß treten' (вт 9о3) benutzt, das auch im Althochdeutschen und Altniederländischen vorkommt:44

for ðon englum his onbead bi ðe ðcet hie hiolden ðec in allum wegum ðinum. (12) in hondum beorað ðec ne cefre otspurne to stane fot ðine (Vesp.); fordon englum his bebead be đe đaet hie heolden đe on eallum wegum đinum (12) on handum beorad de dy laes cefre cetspurne to stane fot dinne (Junius); WPs. Deut. 32,15. 
[ ] englum his he bebead be ðe pret hy healden pe on eallum wegum pinum. (12) on handum hy berað ðe pylces cefre pu cetsporne cet stane fot pinne (Regius);

forpon englum his he bebead be pe peet hi heoldon pe on eallum wegum pinum. (12) on handum hi berað pe ne cefre pu oðspornyst to stane fot pin (Cambridge);

forpi pe his englum he bebead be pe pret hig healdan pe on eallum pinum wegum. (12) on handum hig berað pe pe loes pu cetsporne to stane pinne fot (Lambeth);

forpam pe englum his god bebead be [ ] peet hy healdon pe on eallum wege pinum. (12) [ ] handum hy berað pe pelces pu ofspurne cet stane [ ] pine (Vitellius);

forðon englas his he bebead be pe pcet hi healdon pe on eallum wegum pinum (12) on handum hi berap pe pilces pu cetsporne cet stane fot pinne (Arundel);

forðon cenglas his bebead be pe peet hi healdon pe on eallum wegum pinum (12) on handum hi berað pe pelces pu cetspurne cet stane fot pinne (Stowe);

[ ] englum his bebead be pe paet hy healden pe on eallum wegum pinum. (12) on handum hy berað pe [ ] pu opsporne cet stane fot pinne (Tiberius); forpam englas his he bebead of pe pcet hi healdan pe on eallan wegan pinan. (12) on handan berap pe palces pu cetsporne cet stane fot pinne (Salisbury); Forpan englum his bebead be ðe ðette gehealdon pe on eallan wegas pine (12) On handum berað pe peet nefre atsporna on stane fot pinum (Canterbury); for pon he his englum bebead, peet hi mid earmum / pe on heora handum heoldan georne, / pcet pu on wil-wega wealdan mostest. (12) And pe on folmum feredan swylce, / pe laes pu frcecne on stan fote spurne. (Paris).

Das Verb spornan ist gemeingermanisch mit Ausnahme des Gotischen, vgl. Kroonen 2013: 471. Auch hier fällt wieder auf, dass die altenglischen Psalter alle praktisch denselben Text haben.

24. Ps. 100,7: NON HABITABAT IN MEDIO DOMUS MEÆ QUI FACIT SUPERBIAM 'keiner wird wohnen mitten in meinem Haus, der Hochmütigkeit pflegt', wobei habitabit in einigen Handschriften eine Variante ist. Gotisch: ni gabauip in midjamma garda meinamma taujands hauhairtein in Bologna, Bl. IIr.14-15. Got. gabauip weicht von gr. katôkei (Imperfekt von katoikéô) ab, indem das Verb durch das Präfix einen perfektiven Charakter bekommt und somit wohl das Futurum ausdrückt. In der lateinischen Überlieferung sind in dieser Stelle verschiedene Tempora belegt (Falluomini 2014: 293). Auch hier könnte Einfluss des Lateinischen oder eine griechische Variante vorliegen. Die Verbindung in midjamma garda meinamma ist wohl eine gotische Konstruktion, wie Falluomini (2014: 
293) andeutet. Sie entspreche der Konstruktion in midjaim laisarjam in Luk. 2,46. Das Substantiv hauhhairteins 'Hochmut' ist vermutlich eine Lehnübersetzung. Im Althochdeutschen hat Notker Folgendes: In mînemo hérzen nebúet. der úbermuôte ist. Hier interpretiert Notker den Ausdruck 'mein Haus' als 'mein Herz', wie in den lateinischen Kommentaren zu den Psalmen gemacht wird: "Refer te ad domum illam id est, ad cor" (Notker Latinus). Die frühmittelhochdeutschen Psalter übersetzen buchstäblich: in mitten huses mines (Windb.) und almitten huses mines (Vind.), vgl. Leningrad: in midden mijns huus. Im Altenglischen finden sich:

ne eardað in midle huses mines se doeð oferhygde (Vesp.); ne eardađ on midle huses mines se đe doeđ oferhygde (Junius); [ ] eardap on middele huses mines se pe dep ofermodnesse (Regius); ne eardceð on midle huss minys se deð ofyrhygde (Cambridge); ne geeardað t. ne wunap on midlunge mines huses se pe deð modignysse (Lambeth); 45 ne eardað on midele huses mines se ðе deð ofermodignes (Vitellius); na eardap on middele huses mines se pe dep ofermodnesse (Arundel); na eardað on middele huses mines se ðe dep ofermodignysse (Stowe); [ ] eardað on middele huses mines se pe deð ofermodnesse (Tiberius); fehlt (Salisbury);

Ne ecerdcep on middcen hus min se dep oferhid (Canterbury); Ne eardað on midle mines huses, / pe ofer-hygd up ahebbe. (Paris).

Sie gebrauchen im ersten Teil des Verses für lat. habitare statt wonon (Vind., Len., aber auch als Variante in Lambeth) oder bûon (Notker, Windb. und Gotisch) eardian 'wohnen, leben' (вт 232), ein Verb, das auch einmal in den WPs. in derselben Bedeutung erscheint: ordon (l. *ardon) (Ps. 14,1), das im Althochdeutschen jedoch normalerweise 'den Boden bearbeiten, pflügen' bedeutet, aber, namentlich in fränkischen Texten, auch in der Bedeutung 'wohnen' vorkommt (EWA I,350) ebenso wie im altsächsischen 'Heliand' (Tiefenbach 2010). Der gotische Text benutzt im allgemeinen ähnliche Vokabeln wie die anderen germanischen Sprache mit der Ausnahme des Verbs taujan 'tun', aber das ist zu erwarten, da das Verb dōn im Gotischen nicht (mehr?) vorkommt. Für superbia erscheint ae. oferhygd 'Hochmut' (вт 735) in den ältern Psaltern und ofermód (вт 736) und Ableitungen davon in den jüngeren, vgl. Notker und Windb. 
25. Ps. 105,47: SAlvos FAC NOS DOMINE DEUS NOSTER ET CONGREGA NOS DE NATionibus 'errette uns, Herr, unser Gott, und sammle uns aus den Völkern' (Vulgata), vgl. Itala: SALVA NOS DOMINE DEUS NOSTER ET CONGREGA NOS DE GENTIBUS, Im Gotischen findet sich: nasei unsis frauja gup unsar,[jah galis unsis u]s piudom in Bologna, Bl. 1r, Z. if. Es fehlt ein Teil des Textes in der Handschrift. Das griechische Verb an dieser Stelle lautet episunágage und dieses Verb wird Mark. 13,27 von Wulfila mit galisan übersetzt. Ein Imperativ galis unsis passt ausgezeichnet in den Raum auf Zeile 2 des Fragments, sodass die Emendation von Roland Schuhmann (vgl. Falluomini 2017: 286) naheliegt. Das Verb galisan gehört zu *lisan 'sammeln'. Das Verb hat Parallelen - manchmal mit der jüngeren Bedeutung 'lesen' - in allen anderen germanischen Sprachen (Kroonen 2013: 331-32). Notker hat in dieser Stelle: Duô unsih keháltene truhten Got únser [...] unde sámeno unsih fone allen diêten. ze eínemo christiano populo (mit der Glosse liûte 'populo'). Hier wurde das Verb (gi) samnon 'sammeln' benutzt, da im Althochdeutschen das Verb lesan vermutlich an erster Stelle schon 'lesen' bedeutete. Ähnliches findet man in Vind., Windb. und Len. Dasselbe gilt wohl auch für das Altenglische:

hale usic doa dryht [en] god ur 7 gesomna usic of cneorissum (Vesp.); hale us dó dryhten god ure 7 gesomna us of cneorissum (Junius);

hale us do [ ] 7 gesomna us of cynnum (Regius);

hale us do drihtyn god ure 7 gesamna us of cneoryssum (Cambridge);

hale do us drihten god ure 7 gesomna l. gegadera us of elðeodigum (Lambeth);

hale do us driht [en] god ure 7 geso[m]na us of cynnum (Vitellius);

hale do us drihten god ure 7 gesomna us of cynnum (Arundel);

gehcel us driht [en] god ure 7 gesomna us of cynnum (Stowe);

hale do us driht [en] god ure 7 gesomnunga us of cynnum (Tiberius);

hale do us drihten god ure 7 gegadera us of gynne (Salisbury);

hale us dó drihten god ure 7 gesomno us be kennessum (Canterbury);

Do us hale nu, halig Drihten, / and us, se goda God, georne gesamna / of widwegum, per we wean drogað (Paris).

Wieder fällt auf, dass die altenglischen Texte - mit Ausnahme von Paris weitgehend dieselbe Übertragung bieten, vgl. für die Übersetzung von lat. natio Nr. 6. Nur in zwei Fällen, von denen einer als Variante zu gesomna, erscheint gaderian 'versammeln' (Bт 356), eine andere übliche Übersetzung für lat. congregare. 
26. Ps. 109,1:Dixit Dominus Domino meo SEDE A DeXtris meis DoneC PONAM INIMICOS TUOS, SCABILlum PEDUM TUORUM. 'der Herr hat zu meinem Herrn gesagt: "Sitze an meiner Rechten, bis ich deine Feinde zum Schemel deiner Füße mache”. Der Text in Luk. 20,42-43 erwähnt ausdrücklich, dass aus den Psalmen zitiert wird: jah silba Daweid qipip in bokom psalmo〈no $\rangle$ : qap frauja du fraujin meinamma: sit af taihswon meinai, unte ik galagja fijands peinans fotubaurd fotiwe peinaize. 'und David selbst sagt im Buch der Psalmen', wonach der Text zitiert wird. Der Vers wird teilweise auch in Röm. 8,34 zitiert: ... saei ist in taihswon gudis 'der ist zur Rechten Gottes'. Der gotische Text benutzt taihswo 'die Rechte', wie auch die altniederländische Übersetzung macht: theseuun 'dexteræ' (Ps. 19,7) und teseuuon 'dexteram' (Hymn. Ambr. Xxvi,8). Auch das Althochdeutsche kennt zesewa 'die Rechte', vgl. Notker: sizze ze mînero zéseuuun [...] Sízze nében mir. unz ih dîne fîenda tuôie dînen fuôzen. Ne-sint imo alle gentes nu úndertân. die ēr uuâren inimici?, vgl. Sprah der herre. got uater herren minem. gote deme sune sizce ze zeswen minen. Vnze ih gesezce, gelege uiante dine ein scamel fuozze dinere (Windb.). Das Gotische teilt dieses Wort nur mit diesen beiden germanischen Sprachen. ${ }^{46}$ Das Substantiv got. fotubaurd 'Schemel' hat eine exakte Parallele in an. fótborð, aber sonst kommt diese Zusammensetzung nicht vor. Die beiden Elemente sind jedoch allgemein germanisch. Die westgermanischen Texte bevorzugen (fuot)skamel, vgl. WPs. fuotscamel 'scabillum' in Ps. 98,5. Im Altenglischen kommen vor: ${ }^{47}$

cwið dryht [en] to dryht [ne] minum site to ðe swiðran minre oð ðœet ic sette feond Jine scomul fota Jinra. (Vesp.);

[ ] dryhten hlaforde minum site to swidran minre ot dcet ic gesette fiend pine fotscamul fota pinra (Junius);

[ ] drihtne [ ] site of swyprum minum oð ic sette fynd pine fotscamul fota Jinra (Regius);

sæede l cwce drihtyn to drihtne minum site to swipran minre oppcet ic sette fynd pine fotsceamul fota pinra (Cambridge);

scede drihten to drihtne minum site cet swyðrum minum op peet ic asette feond pine on fotsceamele fota pinra (Lambeth);

46 Im Falle des Altniederländischen ist es möglich, dass die Form tesewa aus dem Althochdeutschen stammt oder von ihm beeinflusst wurde, denn in diesem Text erscheint auch forthora in derselben Bedeutung (de Grauwe § 191). 
cwceð drihten drihtene minum site of swyprum minum oð ic [s]ette fynd pine fotscamul fota pinra (Vitellius);

cwcep drihten drihtne minum sitte of swipre minum op ðcet ic sette fynd pine fotscamol fota pinra (Arundel);

scede drihten drihtne mine sitte to swyprum minum oð pcet ic sette fynd pine fotscamul fota pinra (Stowe)

cwceð drihten drihtene minum site of swyðrum minum oð ic sette fynd pine fotseamul fota pinra (Tiberius)

cwed drihten to drihtne minum site on pee swyprcen minre or ic sette fynd pine sccemol fot pinre (Canterbury).

Die altenglischen Texte benutzen also ausnahmslos ae. swîfra 'dexter' (в 959), das nur eine Parallele in as. swidra hand 'rechte Hand' (Tiefenbach 2010: 389) hat.

27. Ps. 111,9: Dispersit dedit pauperibus iUstitia eiUs manet in SÆCUlum SÆCUli. 'er streut aus, gibt den Armen. Seine Gerechtigkeit bleibt ewiglich'. Im Gotischen erscheint: swaswe gamelip ist: tahida, gaf unledaim; uswaurts is wisip du aiwa. 'Wie geschrieben ist, er streute aus; er gab den Armen: seine Gerechtigkeit bleibt ewig' (II Kor. 9,9). Das Verb tahida kommt von tahjan 'ausstreuen', das sonst nur eine Parallele im Altnordischen hat: teeja '(Wolle) karden'. Got. unleps 'arm' und unledi 'Armut' haben nur im Altenglischen Parallelen, z.B. unlced 'arm' (вт 1119). Das Wort uswaurhts 'Gerechtigkeit' hängt mit waurkjan 'tun' zusammen. Notker hat an dieser Stelle: Fone diû spéndota er sin guôt. kab iz armen. Daz sin réht uuéret iêmer. In den 'Lubliner Palmen' fehlt gerade in diesem Vers die interlineare Übersetzung (Tiefenbach 2003: 445). Im Altenglischen erscheinen:

tostregd salde ðearfum rehtwisnis his wunað in weoruld weorulde (Vesp.); tostregde sealde dearfum 7 ryhtwisnes his wunat on weorolda "weorold (Junius);

he dcelde sealde pearfum 7 rihtwisnes his wunad on woruld worulde (Regius);

tostregde sealde pearfum rihtwisnys his wunap on woruld worulde (Cambridge);

he todcelde 7 sealde pearfum rihtwisnesse his wunap on weorlda weorl $[d]$ (Lambeth);

he leedde he sealde pearfum rihtwisnesse his wunað on worulda woruld (Vitellius);

he dcelde sealde pearfum rihtwisnesse his wunap ${ }^{*} 0[n]$ worlda world (Arundel); 
he dcelde sealde pearfa rihtwisnes his wunað on worulda woruld (Stowe); he loedde sealde pearfum rihtwisnesse his wunap on woruld worulde (Tiberius);

dcelde sealde pcerfan rihtwisnesse his wunað on worelde [ ] (Salisbury); Tostencep secelde pecerfeen \& soðfestnes his wunap on worlde ceworlde (Canterbury);

Se pe his cehta ealle tostredeð / and pearfendum pa gedceleð, / his soðfcestnyss wunad symble oð ende; (Paris).

Sie haben für dispersit unterschiedliche Formen: tô-stregdan 'ausstreuen' (вт 1007), dcelan 'verteilen' (вт 194), tô-stencan 'verbreiten' (в Т 1007) und die etwas seltsame Übertragung lcedan 'leiten' (вт 6о8) in Vitellius und Tiberius. Für lat. dare 'geben' haben sie ziemlich einheitlich sellen 'geben' (вт 861-62), s. oben Nr. 21. Auch sonst weisen sie im Großen und Ganzen denselben Text auf. Für lat. in sceculum sæeculi hat auch WPs. 6o,9 dieselbe Übersetzung: an uuerildi uuerldis (s. auch Ps. 9,6), vgl. ahd. in werlt werlte (Schützeichel 2006: 406).

28. PS 117,22: LAPIDEM QUEM REPROBAVERUNT ÆDIFICANTES FACTUS EST IN CAPUt ANGULI. (23): A DoMino FACTUM EST ISTUd HOC ${ }^{48}$ EST MiRABIle IN OCULIS Nostris. 'Der Stein, den die Bauleute abgelehnt haben, ist hier zum Eckstein ${ }^{49}$ gemacht worden. (23) Dies wurde vom Herrn getan und ist ein Wunder für unsere Augen.' Gotisch: nih pata gamelido ussuggwup: stains pammei uswaurpun pai timrjans, sah warp du haubida waihstins? fram fraujin warp sa jah ist sildaleiks in augam unsaraim. 'Und habt ihr nicht in der Schrift gelesen; Der Stein, den die Bauleute ablehnten, ist zum Haupt der Ecke geworden: Dies hat der Herr getan und ist wunderbar in unseren Augen' (Mark. 12,10-11). Ein Teil dieses Psalmes wird auch Luk. 20,17 zitiert: appan hva ist pata gamelido: stains pammei uskusun pai timrjans, sah warp du haubida waihstins? 'Was ist es denn, das geschrieben worden ist: Der Stein, den die Bauleute verwarfen, dieser ist zum Haupt der Ecke geworden?' Das Verb ussiggwan 'lesen, rezitieren' scheint im Gotischen spezifisch für das Lesen und Rezitieren der Psalmen gebraucht zu werden. ${ }^{50}$ Weiter fällt auf, dass die zwei Stellen in Wulfilas Text unterschiedliche Verben benutzen: uswairpan 'hinauswerfen' und uskiusan 'ablehnen, verwerfen', obwohl der

48 Statt hoc findet sich in manchen Texten et, wie auch in einigen altenglischen Versionen.

49 Die Stelle, die im Lateinischen als 'Haupt der Ecke' übertragen wird, ist eine buchstäbliche Übersetzung desselben hebräischen Ausdrucks. Es kann auch als 'Eckstein' übertragen werden'.

Vgl. Bologna: in pamma faura suggwanin liupa das sich auch auf diesen Psalmvers bezieht. 
griechische Text ein und dasselbe Verb verwendet: $\dot{\alpha} \pi \varepsilon \delta 0 x i \mu \alpha \sigma \alpha v$. Notkers Text hat: Der steîn den iudei zimberon ferchúren. der uuard ze hoûbete des uuíncheles. daz chît ze houbetstêine. Fóne Góte uuard daz. Vnde in unseren ougon ist iz uuunderlih. Er verwendet also ein ähnliches Verb wie der gotische Text in Luk.: firkiosan 'ablehnen, verachten', ebenso wie Windb.: Den stein den die uerchuren die zimberente der worden ist an daz houbet des wincheles. (23) Vone deme herren getan ist dizce daz ist wunderlih in ougen unseren, während Vind. gerade das andere Verb gewählt hat: und den stein die da uerwurfen die murcere der worden ist in daz hovbet des ekkes (23) uon got getan ist dice daz ist wunterlich in ovgen unseren (Vind.). ${ }^{51} \mathrm{Im}$ Altenglischen findet man folgende Texte:52

stan ðone widcurum timbrende des geworden is in heafud hwommes (23) from dryht [en] geworden is 7 is wundurlic in egum urum (Vesp.);

stan done widcuron đa timbrendan des geworden is on heafod hwammes (23) from dryhtne geworden is 7 is wundurlic in eagum urum (Junius);

stan pone hy wipcuron timbriende pes geworden is on heafoud hyrnan (23) fram drihtne gedon is 7 is wundurlic on eagum urum (Regius);

stan pœene wiðcurun timbriynde pes gewordyn ys on heafud hwommys (23) from dryhtne gewordyn ys 7 ys wundurlic in egum urum (Cambridge); stan pone pe hy gefandedon l. pone pe awurpon ða timbriendan pes geworden is on heafde hyrnan (23) fram drihtne geworden is pis 7 is wundorlic on eagum urum (Lambeth);

[ ] pone hy wiðcuron timbrigende pes geworden is on heofod hyrnan (23) fram driht [en] geworden is [ ] is is wundorlic on eagum urum (Vitellius); stan pone hi wipcuron pa timbrigendan pes geworden is on heafod hirnan (23) fram drihtne gedon is peet 7 is wundorlic on eagum hurum (Arundel); stan pone hi wiðcuron pa tymbrigendan pes geworden is on heafod hyrnan (23) fram drihtne geworden is peet peet is wundorlic on eagum urum (Stowe);

stan pone hi wipcuran timbriende pes gedon is on heauod hyrnan (23) [ ] gedon is [ ] wunderlic on egan ure (Salisbury);

Stcen pone hie costodon tymbrodon pes geworden is on heafod on whermme from drihtne geworden is 7 is wundor on ecegcen urum (Canterbury);

$5^{1} \quad$ Vgl. auch Len.: Die steen, die de temmerlieden verworpen, die es gemaect int hoeft van den houke. (23) Van den here es dit geschiet ende es wonderlijc in onzen ogen.

Der Text fehlt in Tiberius. 
Pone sylfan stan pe hine swyðe cer / wyrhtan awurpan, nu se geworden is / hwommona heagost; halig Dryhten / to wealles wraðe wis teofrade; / pcet is urum eagum eall wundorlic. (Paris).

Im Altenglischen findet man also für lat. reprobare dieselben zwei Verben wie im Gotischen und im Deutschen. Lat. cedificare wird überall, wie bei Notker, mit dem Verb 'zimmern' übertragen. Für den Begriff 'Ecke' verwenden die altenglischen Texte hwamm (вт 572) und hyrne (вт 583), wobei ersteres Wort nur altenglisch zu sein scheint, während das zweite Paralellen im Altfriesischen (herne) und Verwandte (horn 'Horn; Spitze, Ecke') in den anderen germanischen Sprachen hat.

29. Ps. 117,26: Benedictus Qui venturus est ${ }^{53}$ in nomine Domini: 'Gesegnet ist der, der kommt im Namen des Herrn..54 Auf Gotisch: osanna, piupida sa qimanda in namin fraujins! 'Hosanna; gesegnet ist derjenige, der kommt im Namen des Herrn' (Mark. 11,9). Dieselbe Stelle erscheint zum Teil auch in Luk. 19,38: qipandans: piupida sa qimanda piudans in namin fraujins und in Joh. 12,13: jah hropidedun: osanna, piupida sa qimanda in namin fraujins, piudans Israelis. Für piupjan 'segnen' siehe Nr. 2 oben. Notker: Ze guoto genamdo der in truhtenes namen chumet. [...] Vuir ségenoton iûh uzer demo Gótes hûs, vgl. damit: gewihter. wole gesprochener du der chumftich bis in dem namen gotes (Windb.). Die altenglischen Psalter gebrauchen hier wieder ausnahmslos Formen des Verbs bletsian 'segnen' (BT 109):

gebledsad se ðe cwom in noman dryht [nes] (Vesp.); sie gebletsod se đe com on naman dryhtnes (Junius); gebledsod se pe com on naman drihtnes (Regius); gebledsod se ðe com on naman drihtnys (Cambridge); gebletsod se ðe com on naman drihtnes (Lambeth); gebletsod se ðe towerd is [ ] naman driht [enes] (Vitellius); gebletsod sy se pe toweard is on naman drihtnes (Arundel); gebletsod pe toweard is on naman drihtnes (Stowe); gebletsod se pe com on naman drihtenes (Salisbury); gebletsce se ðe com on nomøen drihtnes (Canterbury); Gebletsad is, se pe com ofer bearna gehwylc / on Dryhtnes naman dcedum marum. (Paris). etwa bei Notker.

54 Vgl. auch Mark 11,10: piupido so qimandei piudangardi in namin 〈fraujins〉. 
Im Großen und Ganzen haben die altenglischen Übersetzungen wieder denselben Text. Die Varianten beim Verb hängen wohl mit den Varianten im lateinischen Text zusammen.

Auffällig ist, dass die altenglischen Übertragungen sehr oft einen ähnlichen Text haben. Dies hängt zum Teil wohl zusammen mit den ziemlich engen Verbindungen zwischen den einzelnen Texten, s. Berghaus 1979. Für das Gotische kann man feststellen, dass Wulfila bei abstrakten Begriffen offenbar eigene Wege ging. In einigen Fällen wurde im Gotischen eine möglicherweise archaische Form beibehalten. Die kontinentalen Texte weisen - insofern sich das bei der beschränkten Anzahl Textzeugen sagen lässt - im allgemeinen eine mehr unterschiedliche Wortwahl auf.

\section{Literatur}

Baesecke, Georg, 1930. Der deutsche Abrogans und die Herkunft des deutschen Schrifttums, Halle/Saale. Neudruck: Hildesheim-New York 1970.

Berghaus, Frank-Günter, 1979. Die Verwandtschaftsverhältnisse der altenglischen Interlinearversionen des Psalters und der Cantica (Palaestra 272). Göttingen.

Blom, Alderik H., 2017. Glossing the Psalms. The emergence of the written vernaculars in Western Europe from the seventh to the twelfth century. Berlin-Boston.

Brenner, E. (Hg.), 1909. Der altenglische Junius-Psalter. Heidelberg.

в $=$ Bosworth, J./Toller, T. N. An Anglo-Saxon Dictionary, based on the manuscript collections of the late Joseph Bosworth. Neudruck: Oxford 1976.

вт Suppl. $=$ Northcote Toller, T. An Anglo-Saxon Dictionary, based on the manuscript collections of the late Joseph Bosworth. Supplement, Oxford 1908-1916.

Campbell, A. P. (ed.), 1974. The Tiberius Psalter, edited from British Museum ms Cotton Tiberius (Ottawa Mediaeval Texts and Studies II). Ottawa.

EWA = Lloyd, Albert L. \& Springer, Otto \& Lühr, Rosemarie(Hgg.), 1988-... Etymologisches Wörterbuch des Althochdeutschen. Band I-.... Göttingen-Zürich.

Falluomini, Carla, 2014. "Zum gotischen Fragment aus Bologna”, in: ZfdA 143, 281-305.

Falluomini, Carla, 2017. "Zum gotischen Fragment aus Bologna II: Berichtigungen und neue Lesungen", in: ZfdA 146, 284-294.

Finazzi, Rosa Bianca \& Tornaghi, Paola, 2013. "Gothica Bononiensia: Analisi linguistica e filologica di un nuovo document”, in: Ævvum 87, 113-153.

Gll. = Steinmeyer \& Sievers. 
Grauwe, Luc de, 1979-82. De Wachtendonckse psalmen en glossen. Een lexikologischewoordgeografische studie met proeve van kritische leestekst en glossaria. I-II. Gent.

Harsley, Fred (Hg.), 1889. Eadwine's Canterbury Psalterwith Introduction and Notes from the Manuscript in Trinity College, Cambridge. Part II. Text and Notes (E ETs). London.

Heymans, J. G. (uitg.), 1973. Het Psalter van Leningrad (Verzameling van Middelnederlandse bijbelteksten. Kleine Reeks, Afd. v: Psalters, Deel I). Leiden.

Hofmann, Dietrich \& Popkema, Anne Tjerk, 2008. Altfriesisches Handwörterbuch, Heidelberg.

Kimmens, Andrew C. (ed.), 1979. The Stowe Psalter, Toronto-Buffalo-London.

Kirchert, Klaus, 1979. Der Windberger Psalter. Band I: Untersuchung. Band II: Textausgabe (Münchener Texte und Untersuchungen zur deutschen Literatur des Mittelalters 59). München.

Köbler, Gerhard, 1975. Lateinisch-germanistisches Lexikon (Arbeiten zur Rechts- und Sprachwissenschaft). Göttingen.

Kroonen, Guus, 2013. Etymological Dictionary of Proto-Germanic (Leiden IndoEuropean Etymological Dictionary Series 11). Leiden.

Kuhn, Sherman M. (ed.), 1965. The Vespasian Psalter. Ann Arbor.

Langbroek, Erika, 199o. "Condensa atque tenebrosa; die altfriesischen Psalmen: Neulesung und Rekonstruktion (UB Groningen Hs. 404)", in: Rolf H. Bremmer Jr., Geart van der Meer \& Oebele Vries (Hgg.), Aspects of Old Frisan Philology = ABäG 31/32 \& Estrikken 69. Amsterdam-Groningen, 255-84.

Lehmann, Winfred P., 1986. A Gothic Etymological Dictionary. Based on the third edition of Vergleichendes Wörterbuch der gotischen Sprache by Sigmund Feist. Leiden.

Lexer, Matthias, 1872-78. Mittelhochdeutsches Handwörterbuch. Band I-III, Leipzig.

Lindelöf, U. (Hg.), 19oga. Der Lambeth-Psalter. Eine altenglische Interlinearversion des Psalters in der Hs. 427 der erzbischöflichen Lambeth Palace Library (Acta Societatis Scientiarum Fennicæ. Tom. Xxxv, Nr.1). Helsingfors.

Lindelöf, U. (Hg.), 19ogb. Die altenglischen Glossen im Bosworth Psalter. Helsinki.

Moyise, Steven \& Menken, Maarten J. J. (eds.) 2004. The Psalms in the New Testament (The New Testament and the Scriptures of Israel), London-New York.

Oess, G., 1920. Der altenglische Arundel-Psalter. Heidelberg.

O’Neill, Patrick P., 2016. Old English Psalms. Cambridge.

Orel, Vladimir, 2003. A Handbook of Germanic Etymology. Leiden-Boston.

Pulsiano, Phillip (ed.), 2001. Old English glossed Psalters. Psalms 1-50, published in association with The Centre of Medieval Studies, University of Toronto, Toronto-Buffalo-London.

Quak, Arend, 1975. Wortkonkordanz zu den altmittel- und altniederfränkischen Psalmen und Glossen, Nach den Handschriften und Erstdrucken zusammengestellt (Amsterdamer Publikationen zur Sprache und Literatur 22). Amsterdam. 
Quak, Arend, 1981. (Hg.). Die altmittel- und altniederfränkischen Psalmen und Glossen.

Nach den Handschriften und Erstdrucken neu herausgegeben (Amsterdamer Publikationen zur Sprache und Literatur 47). Amsterdam.

Quak, Arend, 1988. "Zum Paderborner Fragment einer interlinearen altsächsischen Psalmenübersetzung", in: $A B \ddot{a} G$ 26, 1-10.

Roeder, F. (Hg.), 1904. Der altenglische Regius-Psalter. Eine Interlinearversion in Hs. Royal 2.B.5 des Brit. Mus. (Studien zur englischen Philologie, Heft XVIII), Halle a. S.

Rosier, James L. (ed.), 1962. The Vitellius Psalter edited from British Museum Ms Cotton Vitellius E. xviii. Ithaca.

Schützeichel, Rudolf, 2006. Althochdeutsches Wörterbuch. 6. Auflage, überarbeitet und mit den Glossen erweitert. Tübingen.

Sisam, Celia \& Sisam, Kenneth (eds.)., 1959. The Salisbury Psalter. Edited from the Salisbury Cathedral Ms. 150 (Early English Text Society 242). London-New York-Toronto.

Snædal, Magnús. A Concordance to Biblical Gothic, I: Introduction. Texts; II: Concordances. 3rd rev. ed. Reykjavík 2013.

Steinmeyer, Elias \& Sievers, Eduard. (Hg.), 1879-1922. Die althochdeutschen Glossen. I-V. Berlin 1879-1922. Neudruck: Berlin 1968-69.

Steinmeyer, Elias von (Hg.), 1916. Die kleineren althochdeutschen Sprachdenkmäler. Neudruck: Weidmann, Berlin 1971.

Tax, Petrus W. (Hg.), 1952-55. Notker der Deutsche. Der Psalter. (Die Werke Notkers des Deutschen. Bd. 8-10). Halle a.d. Saale.

Tiefenbach, Heinrich, 2003. "Die altsächsische Psalmenübersetzung im Lublin/ Wittenberg Psalter", in: Bergmann, R. (Hg.), Volkssprachig-lateinische Mischtexte und Textensembles in der althochdeutschen, altsächsischen und altenglischen Überlieferung. Mediävistisches Kolloquium des Zentrums für Mittelalterstudien der Otto-Friedrich-Universität Bamberg am 16. und 17. November 2001 (Germanistische Bibliothek Bd. 17). Heidelberg, 385-465.

Tiefenbach, Heinrich, 2010. Altsächsisches Handwörterbuch/A Concise Old Saxon Dictionary. Berlin-New York.

Törnqvist, Nils (Hg.), 1934. Cod. Pal. Vind. 2682. I. Eine frühmittelhochdeutsche Interlinearversion der Psalmen aus dem ehemalige Benediktinerstifte Millstatt in Kärnten zum ersten Male herausgegeben. Lund-Kopenhagen.

Toswell, M. J., 2014. The Anglo-Saxon Psalter (Medieval Church Studies 10). Turnhout. Vinogradov, Andrej \& Korobov, Maxim, 2016. "Gotische Graffito-Inschriften aus der Bergkrim", in: ZfdA 145, 141-57.

Vinogradov, Andrej \& Korobov, Maxim, 2018. "Gothic Graffiti from the Mangup Basilica", in: Advances in Gothic Philology and Linguistics. NOWE LE 71, 223-35. 
Vulgata = Biblia sacra iuxta vulgatam versionem adiuvantibus Bonifatio Fischer OsB, Iohanne Gribomont OsB, H. F. D. Sparks, W. Thiele recensuit et brevi apparatu instruxit Robertus Weber OsB. I-II. Stuttgart 1969.

Wadstein, Elis (Hg.), 1899. Kleinere altsächsische sprachdenkmäler, mit anmerkungen und glossar (Denkmäler hg. v. Verein für niederdeutsche sprachforschung VI), Norden-Leipzig.

Wiesenekker, Evert, 1991. Word be worde, andgit of andgite. Translation performance in the Old English interlinear glosses of the Vespasian, Regius and Lambeth psalters. Diss. vu Amsterdam. Huizen.

Wildhagen, Karl (Hg.), 1910. Der Cambridger Psalter (Hs. Ff. 1. 23 Univerity Libr. Cambridge) zum ersten Male herausgegeben mit besonderer Berückiichtigung des lateinischen Textes (Bibliothek der angelsächsischen Prosa VII). Hamburg. Neudruck: Darmstadt 1964. 